\title{
Pruebas de tensión al sistema bancario boliviano
}

\section{Stress Testing for the Bolivian Banking System}

\author{
Ignacio Garrón Vedia* \\ Javier Aliaga Lordemann**
}

\section{Resumen}

En términos de regulación, Basilea III insta a los organismos supervisores a avanzar hacia un esquema de supervisión bancaria más orientado al riesgo, especialmente generar medidas o modelos que puedan anticipar o mostrar vulnerabilidades de las entidades financieras. En este marco, en la última década se registró un importante desarrollo de los modelos de macro stress testing, propiciados en primera instancia por el Fondo Monetario Internacional y posteriormente por distintos bancos centrales. El presente documento, a partir de un análisis de escenarios para Bolivia y un conjunto de metodologías de tensión desarrolladas por Cihak $(2001,2004,2005,2007)$ y Blaschke et al. (2001), propone un análisis de los principales riesgos, vinculados a movimientos del tipo de cambio y tasa de interés, que deben gestionar las entidades financieras bolivianas en cuatro áreas fundamentales: riesgo de crédito, riesgo de tasas de interés, riesgo cambiario y riesgo de liquidez.

Los resultados muestran que el sistema bancario tiene una mayor vulnerabilidad a variaciones de las tasas de interés, debido al descalce de plazos existente por los desincentivos generados por los bancos (tasas de interés pasivas bajas para depósitos a plazo fijo). Asimismo, el riesgo de tasas de interés genera el mayor impacto en el CAP en los escenarios planteados,

\footnotetext{
* Investigador del Instituto de Investigaciones Socio Económicas de la Universidad Católica Boliviana San Pablo. Contacto: ignaciogarron@gmail.com

** Director del Instituto de Investigaciones Socio Económicas de la Universidad Católica Boliviana San Pablo. Contacto: jaliaga@ucb.edu.bo
} 
seguido por el riesgo de tipo de cambio y el riesgo crediticio. Por último, los resultados favorables de los stress tests de liquidez reflejan los altos niveles de liquidez con los que cuenta actualmente el sistema bancario.

Palabras clave: Pruebas de tensión, estabilidad financiera, macroprudencial.

\section{Abstract}

In terms of regulation, Basel III urges supervisors to move towards a system of banking supervision more risk-oriented, especially generating measures or models that can anticipate or show vulnerabilities of financial institutions. In this attempt, throughout the last decade there was an important development of macro stress testing models, brought about primarily by the International Monetary Fund and subsequently by various central banks. This paper, based on an analysis of scenarios for Bolivia and a set of stress methodologies developed by Cihak (2001, 2004, 2005, 2007) and Blaschke, Jones, Majnoni y Martínez Peria (2001), proposes an analysis of the principal risks, linked to changes in interest rates and exchange rates, to be managed by Bolivian financial institutions for 2011 set in four key areas: credit risk, interest rate risk, foreign exchange risk and liquidity risk.

The results demonstrate that the banking system is more vulnerable to changes in interest rates due to maturity gap of existing disincentives generated by the banks (low borrowing rates for fixed deposits). In addition, the interest rate risk generates the largest impact on the CAR in the scenarios presented, followed by the exchange rate risk and credit risk. Finally, the favorable results of the liquidity stress tests are explained by high levels of liquidity that the bolivian banking system receantly counts.

Keywords: Stress Tests, Financial Instability, Macroprudential

Clasificación / Classification JEL: E30, E44, G10, G28.

\section{Introducción}

De acuerdo a Díaz (2012), las entidades que conforman los sistemas financieros cumplen cinco roles fundamentales en la economía: (i) gestionan o administran riesgos; (ii) asignan recursos; (iii) procesan información y supervisan a los prestatarios; (iv) canalizan el ahorro de agentes superavitarios a agentes deficitarios de fondos; $y$ (v) facilitan el intercambio de bienes 
y servicios (proporcionan el acceso a los sistemas de pagos). Estas funciones son importantes para el desenvolvimiento de una economía debido a la relación positiva entre crecimiento económico y desarrollo de la actividad de intermediación financiera.

En términos de la supervisión bancaria, uno de los principios básicos enunciados en el Acuerdo de Basilea II es que los bancos deberían contar con un proceso para evaluar la suficiencia de capital, en función del perfil de riesgos de la institución. En este sentido, resulta fundamental tener conocimiento de la estructura de riesgos del sistema bancario boliviano, a efectos de evaluar correctamente los requerimientos de capital.

Asimismo, Basilea II insta a los organismos supervisores a avanzar hacia un esquema de supervisión bancaria orientado al riesgo. Por lo tanto, los modelos de evaluación de riesgos, especialmente las pruebas de tensión, resultan de gran utilidad, toda vez que permiten plantear escenarios hipotéticos, históricos o supuestos de proyección de los factores de riesgo que inciden en el balance bancario.

A nivel de las entidades financieras, es crucial tener conocimiento del perfil de riesgos que enfrentan, para así poder gestionarlos y controlarlos, para lo cual es necesario identificarlos y medirlos. En el estudio bajo análisis se utilizó la herramienta stress testing (ST) para medir los riesgos, de manera de evaluar tanto la solidez como las amenazas que enfrenta el sistema financiero boliviano, así como la capacidad de resistencia de las entidades bancarias ante choques adversos.

La base metodológica de esta herramienta utiliza el diseño de escenarios hipotéticos y/o históricos que permiten simular situaciones extremas. Las pruebas de tensión analizan situaciones poco probables pero factibles que contengan factores de riesgo para la macroeconomía de un país. Los resultados generalmente se presentan en términos de pérdidas para las entidades financieras y el eventual deterioro en su solvencia, medida a través del Coeficiente de Adecuación Patrimonial (CAP) ${ }^{1}$.

El presente documento, a partir de un análisis de escenarios y aplicando un conjunto de metodologías, propone un análisis de los principales riesgos que deben gestionar las entidades financieras bolivianas al año 2011, mediante la herramienta de ST, enmarcado en cuatro áreas

$1 \quad C A P=\frac{\text { Patrimonio neto }}{\text { Activos ponderados por riesgo }}$ 
fundamentales: riesgo de crédito, riesgo de tasas de interés, riesgo cambiario y riesgo de liquidez.

\section{Antecedentes}

Las captaciones y las colocaciones mantuvieron el elevado ritmo de crecimiento observado en los últimos años, llegando a Bs. 45,910 millones y Bs. 61,223 millones a 2011, respectivamente (véase Gráfico 1). Asimismo, la cartera creció 25\% y los depósitos, 19\%, con relación a 2010, mostrando indicadores superiores al crecimiento de la economía (5.20\%). El crecimiento se ha intensificado entre los años 2007 y 2010 debido al mayor uso de créditos por parte del sector microempresario e informal (Banco Central de Bolivia, 2011).

El crecimiento de los depósitos ha experimentado tasas bastante altas y de hecho superiores al crecimiento de la cartera crediticia, mostrando al sistema bancario boliviano como sólido y coadyuvando a la confianza del público en el sistema financiero, según varias instituciones que analizan la economía boliviana (Banco Central de Bolivia, 2011). El crecimiento de los depósitos se debe a: i) un mayor crecimiento económico en los últimos años (nos hallamos en una fase expansiva del ciclo económico) y ii) un entorno macro favorable, que se reflejço en un mayor crecimiento del PIB. Además, el crecimiento de remesas de los migrantes bolivianos en países extranjeros y el alza de los precios de materias primas, de los cuales Bolivia es exportador, han fomentado una mayor existencia de dinero en la economía, lo cual ha contribuido al alza de depósitos bancarios.

Asimismo como se aprecia en el Gráfico 1, las utilidades anualizadas y el ratio return on equity $\left(\mathrm{ROE}^{2}\right)$ han seguido a partir de 2004 una tendencia positiva. Esto refleja el crecimiento de la cartera y de los depósitos en el último quinquenio, como resultado de la mayor actividad de intermediación financiera, lo que redunda en mayor dinamismo de la economía.

$2 \quad \mathrm{ROE}=$ utilidades anualizadas/patrimonio 
Gráfico 1: Cartera, depósitos, utilidades anualizadas y ROE en el sistema bancario boliviano (en miles de bolivianos)
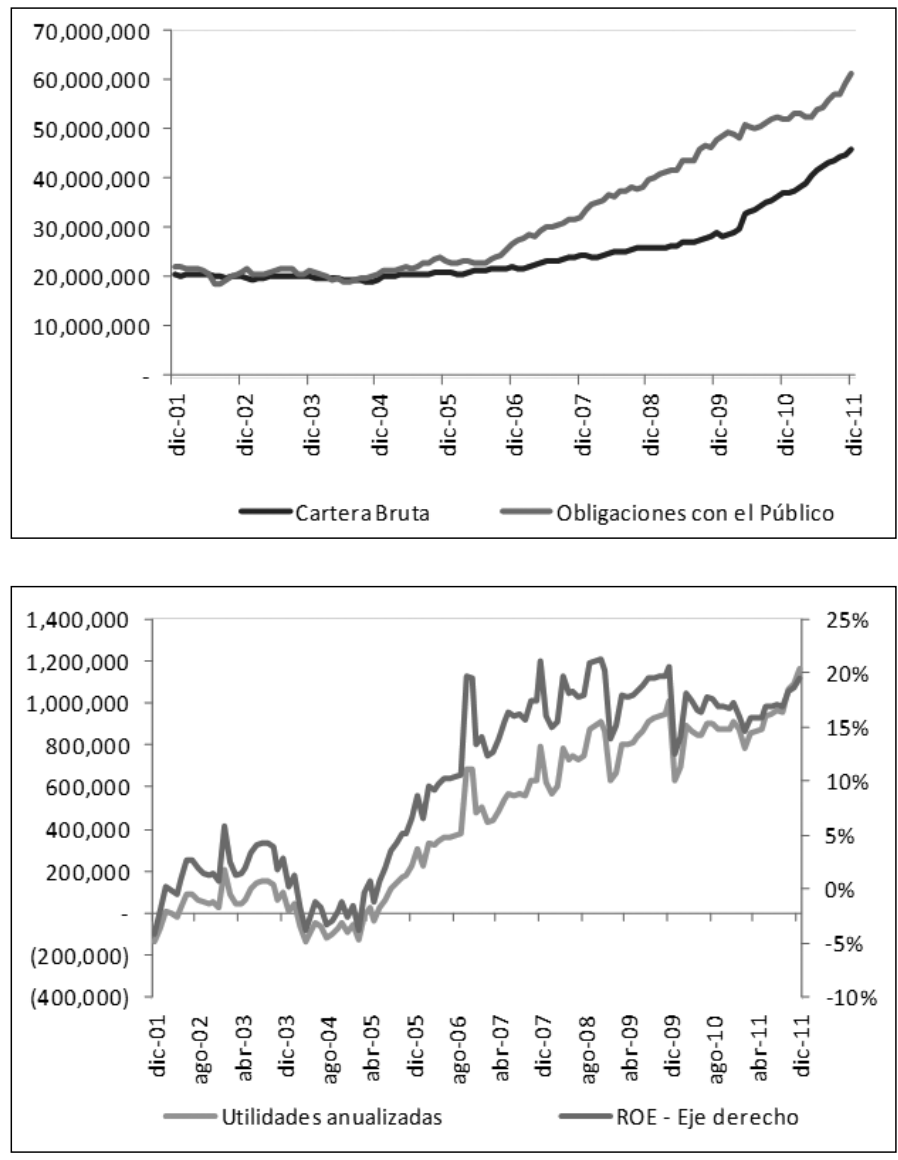

Elaboración propia con datos de la Autoridad de Supervisión del Sistema Financiero.

\section{Los Stress Testing}

En respuesta a la creciente inestabilidad financiera en muchos países en la década de 1990, los hacedores de política se han interesado en comprender mejor las vulnerabilidades de los sistemas financieros y las medidas que podrían ayudar a prevenir las crisis financieras. Una de las principales técnicas para la cuantificación de las vulnerabilidades del sector financiero son los ST. A continuación se muestran dos definiciones importantes: 
El término ST describe un rango de técnicas usadas para evaluar la vulnerabilidad de un portafolio a cambios significativos o excepcionales en el ambiente macroeconómico, pero considerando que estos eventos son plausibles. El objetivo de un ST es mostrar los riesgos de manera más transparente al estimar las potenciales pérdidas en un portafolio en mercados ineficientes. Los ST además son usados para complementar los modelos internos y manejos de sistemas utilizados por las instituciones financieras para decisiones de asignación de capital (Blaschke et al., 2001)

En el contexto del análisis macroprudencial, los ST se refieren a una serie de técnicas estadísticas utilizadas para ayudar a evaluar la vulnerabilidad de un sistema financiero a eventos excepcionales pero plausibles (Cihak, 2004)

La importancia de los ST para el análisis macroprudencial se deriva de la integración de una perspectiva macroeconómica con proyecciones de futuro, un enfoque en el sistema financiero en su conjunto y un enfoque uniforme para la evaluación de la exposición al riesgo a través de los bancos.

A diferencia de los ST realizados a los grandes bancos, los cuales están diseñados para medir la exposición al riesgo de carteras específicas, los ST de todo el sistema aplican un conjunto común de escenarios, basados en una evaluación de los riesgos macroeconómicos y de mercado. Este enfoque uniforme permite la agregación de los resultados, ayudando a identificar las principales vulnerabilidades a nivel de todo el sistema y proporcionar información comparable sobre los perfiles de riesgo a través de los bancos.

\subsection{Estructura del Stress Testing}

El ST presentado en el documento se estructuró en base a cuatro módulos de riesgo individuales: i) riesgo de crédito $^{3}$,ii) riesgo de liquidez, iii) riesgo cambiario, iv) riesgo de tasas de interés, los cuales posteriormente fueron agregados en función de su impacto en el CAP en cada escenario, y a partir de una división de los bancos en función de la nacionalidad de la propiedad accionaria mayoritaria (públicos, privados nacionales y extranjeros).

3 En el presente documento el riesgo de crédito está vinculado con variaciones en tasas de interés y tipo de cambio; sin embargo, éste puede estar vinculado a otras variables. 
Como se aprecia en el Gráfico 2, la transmisión de los choques empieza con la elección de escenarios adversos que reflejan variaciones de la tasa de interés y tipo de cambio. Estas variaciones generan dos efectos:

- Efecto directo: una variación en las tasas de interés produce cambios en el margen financiero y en el portafolio de inversiones de la banca (riesgo de tasa de interés). Asimismo, el efecto de una variación del tipo de cambio plantea cambios en la posición en moneda extranjera (ME) de la banca (riesgo cambiario). La transmisión de estos efectos se muestra en términos del impacto en el CAP. Por último, una variación del tipo de cambio genera variaciones en los depósitos (riesgo de liquidez ${ }^{4}$ ). La transmisión de este efecto se muestra en términos de la liquidez disponible de los bancos al cabo de cinco días.

- Efecto indirecto: implica una variación de la cartera en mora a raíz de una variación en la tasa de interés y tipo de cambio ${ }^{5}$. La transmisión de este efecto se muestra en términos de su impacto en el CAP 6 .

Una vez agregados los impactos de los efectos directo e indirecto a los módulos de riesgo individuales, se procede a mostrar los resultados integrados o agregados, los cuales se refieren a la suma de los impactos producidos en el CAP de los módulos de riesgo de crédito y tasas de interés y cambiario (véase sección 5.1.).

Así también estos resultados se los descompone en tres categorías: bancos privados nacionales, bancos públicos y bancos extranjeros, en función de la concepción de la naturaleza de la nacionalidad de cada banco propuesta por Hurtado, Villacorta y Ferruz (2008).

4 Este efecto se lo relaciona mediante un modelo VEC, planteado en la sección de riesgo de liquidez.

5 La relación entre estas variables fue estimada con un modelo de corrección de errores (VEC), que se lo discute en la sección de riesgo de crédito.

6 El CAP fue seleccionado, ya que engloba al resto de los indicadores microprudenciales bancarios, dado que considera cada uno de los riesgos (crédito, mercado y operativo). Este índice es comparado con el benchmark establecido tanto por la Autoridad de Supervisión del Sistema Financiero (ASFI) y otras instituciones de intermediación financieras de Bolivia (10\%) como por Basilea (8\%), a fin de evaluar la solvencia o la disponibilidad de las instituciones financieras para encarar posibles pérdidas futuras. 
Gráfico 2: Estructura del Stress Testing

Transmisión del impacto:

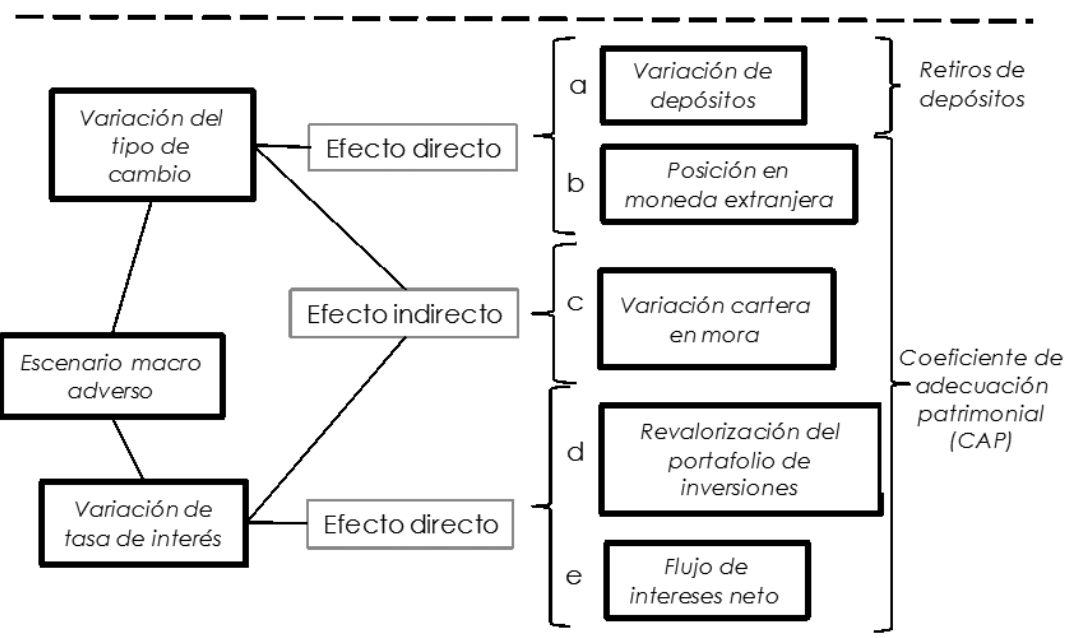

Módulos de riesgo y efectos que incorporan:

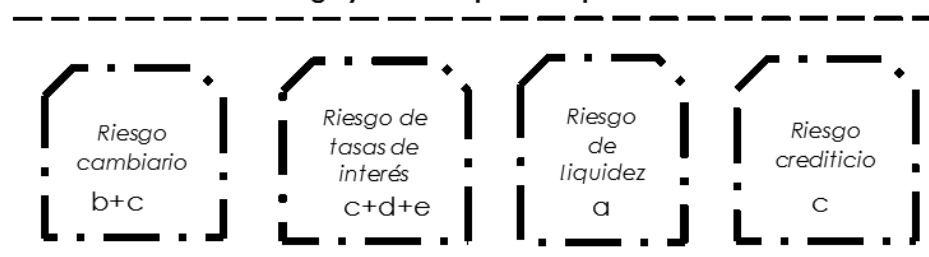

Resultados integrados:

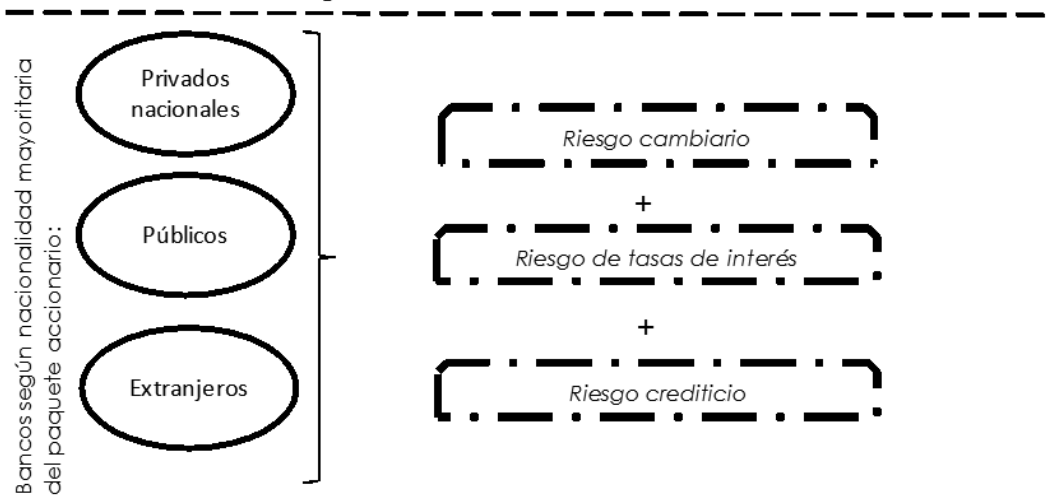

Fuente: elaboración propia 


\subsection{Elaboración del ejercicio de ST}

Existen dos enfoques para la transmisión de choques macroeconómicos y de escenarios propuestos: el enfoque bottom-up, que considera el impacto en cada banco individualmente; y el enfoque top-down, que evalúa la incidencia en las cuentas agregadas del sistema bancario. Cihak (2007) sugiere que la mayoría de los bancos centrales tratan de minimizar los problemas de ambos enfoques ${ }^{7}$ haciendo una mezcla según el riesgo analizado ${ }^{8}$.

\subsection{Formulación de los escenarios}

El aspecto más complejo de las pruebas de estrés es el diseño de los escenarios plausibles, puesto que choques demasiados extremos pueden hacer perder validez al ejercicio. Siguiendo a Blaschke et al. (2001), la formulación de escenarios puede estar basada en: observaciones históricas, que replican algún evento adverso del pasado; simulación Montecarlo, que consiste en identificar algún choque en la cola de la distribución probabilística (por ejemplo, el percentil 95\%); y escenarios hipotéticos, que no tienen una contraparte con la realidad.

A partir de las experiencias del Bank of England (Hoggarth et al., 2004), así como estudios del BIS (Committee on the Global Financial System, 2008) se identificaron los escenarios extremos que tienen una probabilidad de ocurrencia de $0.5 \%$ (nivel de confianza del 99.5\%). En el presente trabajo se llevó a cabo una elección de escenarios históricos, mediante una técnica estadística que permite la identificación de outliers multidimensionales (generados por la combinación de dos series), que se presenta en la sección siguiente.

\section{Metodología}

\subsection{Identificación de datos atípicos}

A partir de las series de datos mensuales de la tasa de interés activa efectiva y el tipo de cambio, en el periodo comprendido entre enero de 1986 y julio de 2012 se procedió a buscar

7 El enfoque top-down tiende a agudizar los efectos agregados en los bancos a nivel individual, mientras que el bottom-up puede conseguir resultados más precisos para capturar los riesgos de liquidez y contagio; sin embargo, este último necesita datos precisos y puede traer problemas en la complejidad de los cálculos.

8 En el presente documento se utiliza el método top-down en los módulos que están vinculados con las variables macroeconómicas (riesgo de crédito, riesgo de tasas de interés y riesgo cambiario), y se aplica la metodología bottom-up en el módulo de riesgo de liquidez que analiza los retiros de depósitos banco por banco en un periodo de cinco días. 
observaciones atípicas (outliers multidimensionales). Son observaciones atípicas, no por el valor que toman en una determinada variable, sino en el conjunto de las variables en un determinado tiempo. Son mucho más difíciles de identificar que los outliers unidimensionales, dado que no pueden considerarse "valores extremos", como sucede cuando se tiene una única variable bajo estudio (McLachlan, 2008). La realización de estos eventos tiene efectos más severos que en el caso unidimensional, ya que distorsionan no sólo los valores de la medida de posición (media) o de dispersión (varianza), sino, muy especialmente, las correlaciones entre las variables.

Para fines de este estudio se propone el uso de la distancia de Mahalanobis como método multidimensional de detección de outliers para encontrar el conjunto de valores de los factores de riesgo (tasas de interés y tipo de cambio), a los cuales se les introducirá el choque para realizar el ejercicio de ST.

\section{Distancia de Mahalanobis}

Este procedimiento consiste en calcular las distancias entre las variables originales, estandarizando los datos de forma multivariada de la siguiente forma:

$$
y=x-\mu \quad ; \quad z=w-\varepsilon
$$

Donde $x-\mu$ se refiere a las variaciones de la tasa de interés centrado a su media $\mu, \mathrm{y}$ $w-\varepsilon$ representa a las variaciones de tipo de cambio centrado a su media $\varepsilon$.

Una vez estandarizados los datos al producto de las desviaciones centradas en sus medias, se le multiplica la inversa de la raíz cuadrada de la matriz de covarianzas de las variables (tipo de cambio y tasa de interés), la cual se representa como $s_{x}^{-\frac{1}{2}}$. La distancia euclidea al cuadrado entre una observación y su media, cero, será como se señala a continuación:

$$
d_{E}^{2}\left(y_{i}, 0\right)=s_{x}^{-\frac{1}{2}}(x-\mu)(w-\varepsilon)=d_{M}^{2}\left(z_{i}, \mu\right)
$$

Con base en esta métrica y utilizando una distribución (Ji cuadrado) con 2 grados de libertad, se detectan los datos atípicos con sus correspondientes probabilidades. La ventaja de este procedimiento es que permite detectar valores atípicos que para el caso de variables correlacionadas mediante un método univariado no podrían ser halladas. Los outliers encontrados al 99.5\% nivel de confianza se muestran en rombos en el Gráfico 3. 
Gráfico 3: Análisis de escenarios (en porcentajes)

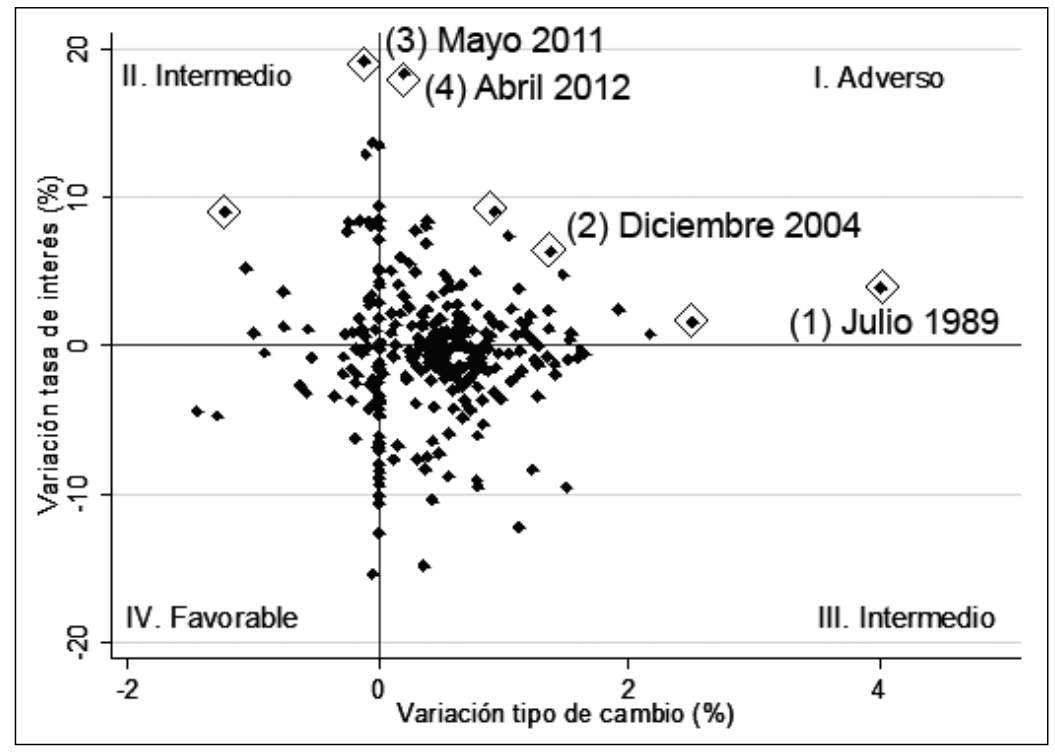

Fuente: Elaboración propia con datos del Banco Central de Bolivia

El Gráfico 3 muestra que los datos atípicos encontrados forman parte de los cuadrantes I y II. Estos cuadrantes fueron definidos en función del efecto negativo en el balance de los bancos, donde una devaluación del tipo de cambio y una variación positiva de la tasa de interés implican un escenario adverso.

De acuerdo a Sheriff (2010), la memoria inflacionaria influye en las decisiones de los agentes económicos, quienes perciben que una mayor depreciación podría tener efectos adversos. Por lo tanto, las depreciaciones tienen un efecto negativo en las entidades de intermediación financieras bolivianas (EIF). Al mismo tiempo, el efecto de la subida de las tasas de interés incide negativamente en la capitalización bancaria, ya que ejerce un efecto negativo en los ingresos financieros (véase sección 4.3.).

Teóricamente, los escenarios que corresponden a un ejercicio de ST son los adversos, con lo cual se seleccionaron los eventos ocurridos en el primer y segundo cuadrante, que coincidieron con el primer escenario del efecto rezagado de la crisis asiática, que generó una desaceleración mundial y una devaluación de los tipos de cambio en América Latina (julio de 1989); el segundo escenario corresponde a la inestabilidad política causada por los conflictos 
durante la etapa final del gobierno de Carlos Mesa Gisbert, que culminó en su renuncia (diciembre de 2004). Adicionalmente se escogieron dos escenarios del cuadrante intermedio, los cuales representan dos eventos que ocasionaron repercusiones importantes en la tasa de interés: el crecimiento inesperado de la demanda de crédito en 10\%, la cual se tradujo en incrementos en la tasa activa de interés (mayo de 2011), y la subida de $24.9 \%$ del encaje legal ${ }^{9}$ en dólares (abril de 2012).

En el Cuadro 1 se exponen las variaciones del tipo de cambio y de las tasas de interés correspondientes a cada escenario, y sus respectivas probabilidades.

\section{Cuadro 1 \\ Escenarios elegidos y su distancia euclidiana}

\begin{tabular}{|c|c|c|c|c|}
\hline \multirow[b]{2}{*}{ Eventos } & \multirow{2}{*}{$\begin{array}{l}\text { Variación de las } \\
\text { tasas de interes } \\
\text { (pp) }\end{array}$} & \multirow[b]{2}{*}{$\begin{array}{c}\text { Variación del tipo } \\
\text { de cambio (\%) }\end{array}$} & \multicolumn{2}{|c|}{ Distancia de Mahalanobis } \\
\hline & & & $\mathrm{d}_{\mathrm{E}}^{2}\left(\mathrm{y}_{\mathrm{i}}, \mathbf{0}\right)$ & $\begin{array}{c}\text { Probabilidad } \\
x^{2}\end{array}$ \\
\hline (1) Crisis asiática & 3.91 & 4.00 & 39.47 & 0.00 \\
\hline (2) Inestabilidad política & 18.33 & 0.20 & 16.85 & 0.00 \\
\hline (3) Shock de créditos & 19.18 & -0.12 & 19.05 & 0.00 \\
\hline (4) Subida del encaje legal & 13.47 & 0.00 & 9.59 & 0.01 \\
\hline
\end{tabular}

Elaboración propia en base a los resultados de la identificación de outliers.

Las variaciones de tasa de interés utilizadas provienen de la serie de la tasa de interés activa en moneda extranjera desde 1986. Esto debido a que la serie de tasas de interés activa en moneda nacional no representa el costo de oportunidad, a pesar de que en los últimos años se ha dado un proceso de bolivianización importante.

Según Sheriff (2010), tanto los prestatarios (instituciones financieras) como los prestamistas (agentes económicos) tienen indexado su costo de oportunidad en dólares, debido a los periodos hiperinflacionarios. De esta manera, las variaciones de tipo de cambio pertenecen al tipo de cambio de compra desde 1986. Es preciso indicar que en ningún de los escenarios se generan variaciones importantes del tipo de cambio, debido a que desde 1986 el Gobierno tiene poder de mercado (venta de divisas), a través del instrumento denominado bolsín. Actualmente, al ser el tipo de cambio fijado discrecionalmente, es improbable una

9 El encaje legal es el porcentaje total de depósitos que un banco, mutual, cooperativa o fondo financiero debe mantener como reserva en el ente emisor. A mayor encaje, las entidades financieras cuentan con menos recursos para expandir su cartera, y generalmente producen mayores tasas activas y/o menores tasas pasivas de aquellos depósitos con mayores tasas de encaje. 
variación abrupta del tipo de cambio. Este régimen de tipo de cambio se denomina crowling peg: mini apreciaciones o depreciaciones no anunciadas. Sin embargo se planteó, un escenario hipotético adicional (depreciación súbita), el cual se refiere a una variación del tipo de cambio de $40 \%$.

\subsection{Riesgo de crédito}

Para analizar los escenarios planteados, se estimó un modelo de corrección de errores (VEC) que relaciona la cartera en mora con variables macroeconómicas; la periodicidad de los datos es trimestral.

\section{Modelo VEC de cartera en mora}

A través de un modelo VEC se relacionço la cartera en mora con variables macroeconómicas, con la finalidad de conocer el origen de las variaciones de la morosidad crediticia. El objetivo es encontrar una relación de equilibrio a largo plazo entre morosidad, actividad económica, tipos de interés y otras variables macroeconómicas relevantes, así como el ajuste de corto plazo entre esas variables por lo que se utilizará la metodología de Johansen (1988).

Las variables utilizadas se eligieron a partir de aproximaciones econométricas similares (Hernández, Valero y Díaz, 2007): Los datos utilizados son de frecuencia trimestral y la muestra comprende el periodo 1998-2011. A continuación se presentan las variables utilizadas:

- Logmora: es la cartera en mora del sistema bancario en logaritmos, suavizada por el método Census X-12; dado su comportamiento estacional (se reduce) en el cuarto trimestre.

- Gcartera: es la primera diferencia de la cartera bruta de los bancos comerciales en logaritmo.

- Dev: es la primera diferencia del tipo de cambio nominal en logaritmo.

- Gpib: es la variación trimestral acumulada del PIB real.

- Tasa: es la tasa promedio de interés activa efectiva en moneda extranjera del sistema bancario boliviano. 
Como se aprecia en el Cuadro 2, las variables resultaron de orden de integración I (1). Los resultados de la prueba de cointegración de Johansen (1988) mostraron que existe al menos una combinación lineal entre estas variables, que da un vector estacionario (véase Anexo 1). La estimación del modelo mostró que las variables son significativas al 10\%, y no se detectaron problemas de autocorrelación ni heteroscedasticidad.

\section{Cuadro 2}

\section{Contraste de raíces unitarias}

\begin{tabular}{ccccccc}
\hline \multirow{2}{*}{ Variables } & \multicolumn{3}{c}{ Niveles } & \multicolumn{2}{c}{ Primeras diferencias } \\
\cline { 2 - 7 } & \multicolumn{2}{c}{ A.Dickey-Fuller } & Phillips-Perron & \multicolumn{2}{c}{ A.Dickey-Fuller } & Phillips-Perron \\
& $P$-Valor & Rezagos & $P$-Valor & $P$-Valor & Rezagos & P-Valor \\
\hline gcartera & 0.4572 & 4 & 0.0170 & 0.0545 & 9 & 0.0001 \\
dev & 0.2568 & 1 & 0.2324 & 0.0000 & 0 & 0.0000 \\
gpib & 0.3436 & 5 & 0.0005 & 0.0000 & 4 & 0.0001 \\
logtasa_act & 0.6049 & 2 & 0.7422 & 0.0001 & 2 & 0.0000 \\
logmora_sa & 0.4338 & 6 & 0.5200 & 0.0000 & 0 & 0.0001 \\
\hline
\end{tabular}

Notas: Los p-valores son de una cola, según MacKinnon. Los rezagos del test ADF se eligieron de acuerdo al criterio de Akaike o Schwarz y viendo que las regresiones auxiliares sean por lo menos ruido blanco (Enders, 2004).

Los signos esperados de los coeficientes de las variables explicativas son los siguientes:

- Tasa de interés activa: signo negativo. Si ocurre un alza de las tasas de interés, se incrementa el costo de los préstamos, lo cual afecta la capacidad de pago de los agentes económicos, que a su vez induciría a un aumento de la cartera morosa.

- Tasa de crecimiento del PIB: signo negativo. Incrementos marginales en la tasa de crecimiento del PIB implican incrementos significativos en el ingreso promedio de las personas en el largo plazo; en este sentido, la capacidad de pago mejoraría y la cartera en mora se reduciría ${ }^{10}$.

- Tipo de cambio: el signo a priori es ambiguo. Una depreciación cambiaria impulsa el crecimiento de las exportaciones, y con ello del PIB, lo que generaría una reducción de la cartera morosa. También se observa que el efecto de la hoja de balance de los clientes

10 Este resultado es intuitivo, debido a que la actividad de intermediación financiera es procíclica. En periodos de expansión económica, existe mayor actividad de intermediación, y debido al mayor ingreso que perciben los agentes económicos, su capacidad de pago mejora, por lo que se esperaría observar menores niveles de morosidad/default. 
deudores del banco (hogares y corporaciones) que dependiendo de la posición neta en divisas que tengan, pudiera mejorar o deteriorar su capacidad de pago ante una eventual depreciación de la moneda ${ }^{11}$.

- Cartera de créditos bruta: signo negativo. En el largo plazo el signo sería ambiguo, dependiendo de si los créditos otorgados fueron productivos o no. En el caso de Bolivia, el mayor porcentaje de créditos se los destina a actividades de comercio, construcción y servicios, principalmente, por lo que es razonable esta aseveración a priori.

$$
\Delta \log \operatorname{mora}_{s a}=-0.063\left(\begin{array}{l}
1 \log \text { mora }_{\text {sat }-1}-10.54 \text { gcartera }_{t-1}-0.28 \text { gpib }_{t-1} \\
+8.831 \text { dev }_{t-1}+1.11 \log \text { tasa }_{a c t-1}
\end{array}\right)
$$

De acuerdo al vector de largo plazo estimado ${ }^{12}$ (1), se halló un efecto negativo de la actividad económica sobre la morosidad de los créditos. Es decir, se verifica el efecto mediante el cual, al incrementarse el ingreso promedio de las personas, su capacidad de pago mejora, lo cual implica una disminución en la cartera en mora.

Asimismo, el efecto de un crecimiento de la cartera bruta disminuye la cartera en mora. Este efecto puede deberse a que los bancos son adversos al riesgo y prefieren prestar con garantías y análisis de riesgo individuales más rigurosas en épocas expansivas.

Por otra parte, fluctuaciones en la tasa de interés y tipo de cambio generan un efecto positivo en la cartera en mora, debido a que la primera encarece el costo del dinero y el segundo, producto de una devaluación, podría deteriorar la capacidad de pago del deudor; además, podría generar expectativas inflacionarias.

\subsection{Riesgo de tasa de interés}

El riesgo de tasas de interés puede descomponerse en dos efectos: indirecto y directo. El primero se refiere a los cambios en la cartera en mora ante variaciones en la tasa de interés. Este riesgo no se expondrá en esta sección, ya que forma parte de la sección anterior (riesgo de crédito).

11 Por ejemplo, una familia que percibe ingresos en moneda nacional y se endeuda en dólares vería mermada su capacidad de pago ante una apreciación del tipo de cambio, lo cual se traduciría en mayores niveles de morososidad.

12 Las variables del modelo resultaron ser significativas al 10\% y los residuos no presentan problemas de autocorrelación ni heteroscedasticidad (véase Anexo 1). 
El riesgo directo puede ser analizado por dos metodologías: El efecto de maduración de brechas, o maturity gap, y el de la duración. La suma de ambos efectos conforma el efecto total de una variación positiva de tasas de interés en un banco. La primera intenta medir el efecto de un aumento en las tasas de interés sobre el margen financiero, y la segunda el efecto de un incremento en las tasas de interés sobre el capital de las EIF.

La metodología de brechas se aplicará en cartera y depósitos, y la metodología de duración, a las inversiones financieras. Asimismo, los efectos anteriormente descritos se expondrán en la sección de resultados en términos de su efecto en el CAP.

\section{Efecto de maduración de la brecha o Maturity Gap}

El maturity gap captura el riesgo de una modificación de las tasas de interés que enfrenta un banco, en términos de flujos, tanto en los ingresos financieros ${ }^{13}$ como en los gastos generados por el pago de los depósitos. Cabe destacar que se asume una variación de 100pb, tanto para tasas activas como pasivas, es decir, se mantiene el spread constante.

Para cuantificar este efecto, se calcula, en primera instancia, la brecha entre la cartera y los depósitos con igual plazo de vencimiento ${ }^{14}$. Posteriormente se introducen choques a las tasas de interés, los cuales afectan directamente a las brechas anteriormente calculadas, y de esta manera se obtiene el cambio en el flujo de intereses netos ante una modificación en las tasas de interés activas y pasivas. Dicho cambio en los plazos está dado por $C_{t}^{\phi}-D_{t}^{\phi}$, y el efecto del cambio en el flujo de intereses netos está dado por:

$$
\Delta \text { Intereses netos }=\sum_{\phi=1}^{t}\left(C_{t}^{\phi}-D_{t}^{\phi}\right) * \Delta i_{t}
$$

Donde:

$C_{t}^{\phi}=$ Cartera crediticia con plazo $\phi$ en el tiempo $t$

$D_{t}^{\phi}=$ Depósitos con plazo $\phi$ en el tiempo $t$

$\Delta i_{t}=$ Cambio en las tasas de interés entre el tiempo ty $(t-1)$

13 Son aquellos que provienen del otorgamiento de créditos.

14 Se calcula la brecha entre la cartera y depósitos para los siguientes plazos de: menor a un año (corto plazo), de uno a cinco años (mediano plazo) y mayores a 5 años (largo plazo), a partir de la información de la página web de la ASFI. 
El mecanismo de transmisión entre el efecto anteriormente expuesto y el CAP es el siguiente: el cambio de los flujos de interés modifica el estado de ganancias y pérdidas, el cual deriva en una alteración directa en el patrimonio neto y por ende se produce una variación del valor del CAP.

\section{Efecto duración o duration effect}

En finanzas, la duración se entiende como la medida de la vida media de un bono. También se la entiende como una aproximación a la relación entre el cambio proporcional del precio del bono y el cambio absoluto de su tasa de rentabilidad (Hull, 2002). En el caso de la banca, en general, la duración de los activos $\left(D_{A}\right)$ es mayor a la duración de los pasivos $\left(D_{P}\right)$, es decir, la brecha entre estos dos será positiva, lo que generará un impacto negativo en la rentabilidad y en el CAP cuando se produce un alza en las tasas de interés (Cihak, 2004 y 2007; Blashke et al., 2001).

Siguiendo la demostración teórica desarrollada por Hernández, Valero y Días (2007) y Cihak (2007), se puede comprobar la anterior aseveración partiendo de la definición de duración de Macaulay. La duración es una medida ponderada de los periodos en que se perciben los flujos, con una ponderación igual al flujo dividido por el precio del instrumento. De esta manera, la duración es definida por la siguiente ecuación:

$$
\text { Duración de Macaulay }=\frac{1^{\prime}}{P}\left[\sum_{t-1}^{N} \frac{t C_{t}}{(1+r)^{t}}+\frac{t * V N}{(1+r)^{t}}\right]
$$

Donde $P$ es el precio del bono, $C_{t}$ el cupón, $V N$ el valor nominal, $r$ el rendimiento y $t$ el tiempo.

Por definición, el precio de un bono es igual al valor presente de sus flujos, donde existe una clara relación negativa entre el precio de un bono y su rendimiento, como se observa a continuación:

$$
P=\sum_{t=1}^{N} \frac{C_{t}}{(1+r)^{t}}+\frac{N * V N}{(1+r)^{N}}
$$


Se puede obtener la siguiente identidad:

$$
\Delta P \cong \frac{-D \Delta r}{(1+r)}
$$

Donde $\Delta r$ representa una variación en la rentabilidad y $\Delta P$ el cambio en el precio del bono. De esta manera, adicionalmente a la definición referida a los plazos promedio descrita anteriormente, la duración aproxima la elasticidad del precio de un instrumento ante cambios en las tasas de interés:

$$
\frac{\Delta A\left(r_{A}\right)}{A\left(r_{A}\right)}=\frac{-D_{A} \Delta r_{A}}{\left(1+r_{A}\right)}, \frac{\Delta L\left(r_{L}\right)}{L\left(r_{L}\right)} \cong \frac{-D_{L} \Delta r_{L}}{1+r_{L}}
$$

Donde $A\left(r_{A}\right)$ y $L\left(r_{L}\right)$ corresponden al valor de los activos y pasivos del sistema bancario, y $r_{A}$ y $r_{L}$ representan a las tasas activa y pasiva, respectivamente. Sustituyendo la diferencia del índice de capitalización (CAP) con respecto a la tasa de interés activa en la anterior ecuación, se obtiene una relación entre la duración y el CAP:

$$
\frac{\Delta\left[C\left(r_{A}, r_{L}\right) / A_{C R}\left(r_{A}\right)\right] A\left(r_{A}\right)}{\Delta r_{A}}=-\frac{\left(L / A_{C R}\right)}{\left(1+r_{A}\right)}\left(D_{A}-D_{L} \frac{1+r_{A}}{1+r_{L}} \frac{\Delta r_{L}}{\Delta r_{A}}\right) \frac{1-\frac{\Delta A_{C R}}{A_{C R}} \frac{C}{\Delta C}}{1-\frac{\Delta A}{A} \frac{C}{\Delta C}}
$$

Donde $C$ corresponde al capital regulatorio y $A_{C R}$ a los activos ponderados por riesgo ${ }^{15}$. Suponiendo que los activos computables $\left(A_{C R}\right)$ varían en igual proporción que los activos totales, es decir que $\Delta A_{C R} / A_{C R} \equiv \Delta A / A$, la ecuación anterior sería la siguiente:

$$
\frac{\Delta\left[C\left(r_{A}, r_{L}\right) / A_{C R}\left(r_{A}\right)\right] A\left(r_{A}\right)}{\Delta r_{A}}=-\frac{\left(L / A_{C R}\right)}{\left(1+r_{A}\right)} G A P_{D}
$$

15 Los activos computables son aquellos activos que se ponderan según la categoría de riesgo en la que se encuentran. La metodología del cálculo se encuentra en la página web de la ASFI, en el documento de Recopilación de Normas del Sistema Financiero, Título IX, Capítulo 8, Sección 4, pág. 89. 
Donde el $G A P_{D}$ quedaría definido como:

$$
G A P_{D}=D_{A}-D_{L} \frac{1+r_{A}}{1+r_{L}} \frac{\Delta r_{L}}{\Delta r_{A}}
$$

Como los bancos y las instituciones financieras, en su mayoría, colocan créditos de mediano o largo plazo con depósitos de corto plazo, ello implicaría que $D_{A}>D_{L}, r_{A}>r_{L}$ y $G A P_{D}>0$. Por lo tanto, la ecuación (8) sugiere que un incremento en las tasas de interés tiene un impacto negativo en el CAP, lo cual representaría una mayor vulnerabilidad del sector financiero.

En Bolivia ${ }^{16}$, el $G A P_{D}>0$; esto se debe a que el $69.8 \%$ de los depósitos ${ }^{17}$ son de corto plazo, es decir, menores a 1 año. En contraste, los créditos tienen un plazo de maduración mayor a 1 año $(76 \%)$, con lo que a priori se intuye el efecto adverso de una subida de tasas de interés en el patrimonio de los bancos.

\section{Descripción del ejercicio en función al impacto en los flujos de interés netos}

Para realizar el ejercicio de riesgo de tasas de interés para el caso boliviano, dado que se deben considerar las cuentas sensibles a modificaciones en la tasa de interés, se estratificaron la cartera, por el lado del activo, y las obligaciones con el público, por el lado del pasivo. Esta división se la realizó de acuerdo a la información de los boletines de bancos de la Autoridad de Supervisión del Sistema Financiero (ASFI), la cual divide la cartera en corto plazo (hasta un año), mediano plazo (de uno a cinco años) y largo plazo (más de cinco años). A partir de esta desagregación se procedió a juntar las cuentas de las obligaciones con el público, con la misma lógica ${ }^{18}$.

Luego de clasificar las partidas del activo y del pasivo por plazo, se obtuvo la brecha, o GAP, entre activos y pasivos, con igual periodo de vencimiento. Cabe destacar que la brecha acumulada es negativa y llega a Bs. 4,470 millones. Este descalce entre los plazos a los que se colocan créditos y a los que se captan fondos podría incrementar la vulnerabilidad del sector

16 Datos obtenidos de los Boletines de Bancos de la ASFI a 201 1; éstos se encuentran en su página web: www.asfi. gob.bo.

17 Sólo se toma en cuenta ahorro y plazo.

18 Esta estratificación no corresponde a la utilizada por la ASFI, ya que esta entidad posee datos de la estratificación por plazo residual (plazos establecidos en el balance tanto para el activo como el pasivo); sin embargo, al ser esta información de carácter no pública, no es posible realizar este cálculo por plazo residual. 
bancario ante un choque en las tasas de interés o de liquidez (corrida de depósitos). Esto es razonable, ya que los bancos no generan incentivos suficientes para que la gente recurra a un depósito a plazo fijo, es decir, las tasas pasivas a 2011 en promedio siguen manteniéndose bajas, aunque muestran algunas mejores (véase Gráfico 4).

\section{Gráfico 4: Evolución de las tasas pasivas y activas en distintas denominaciones}
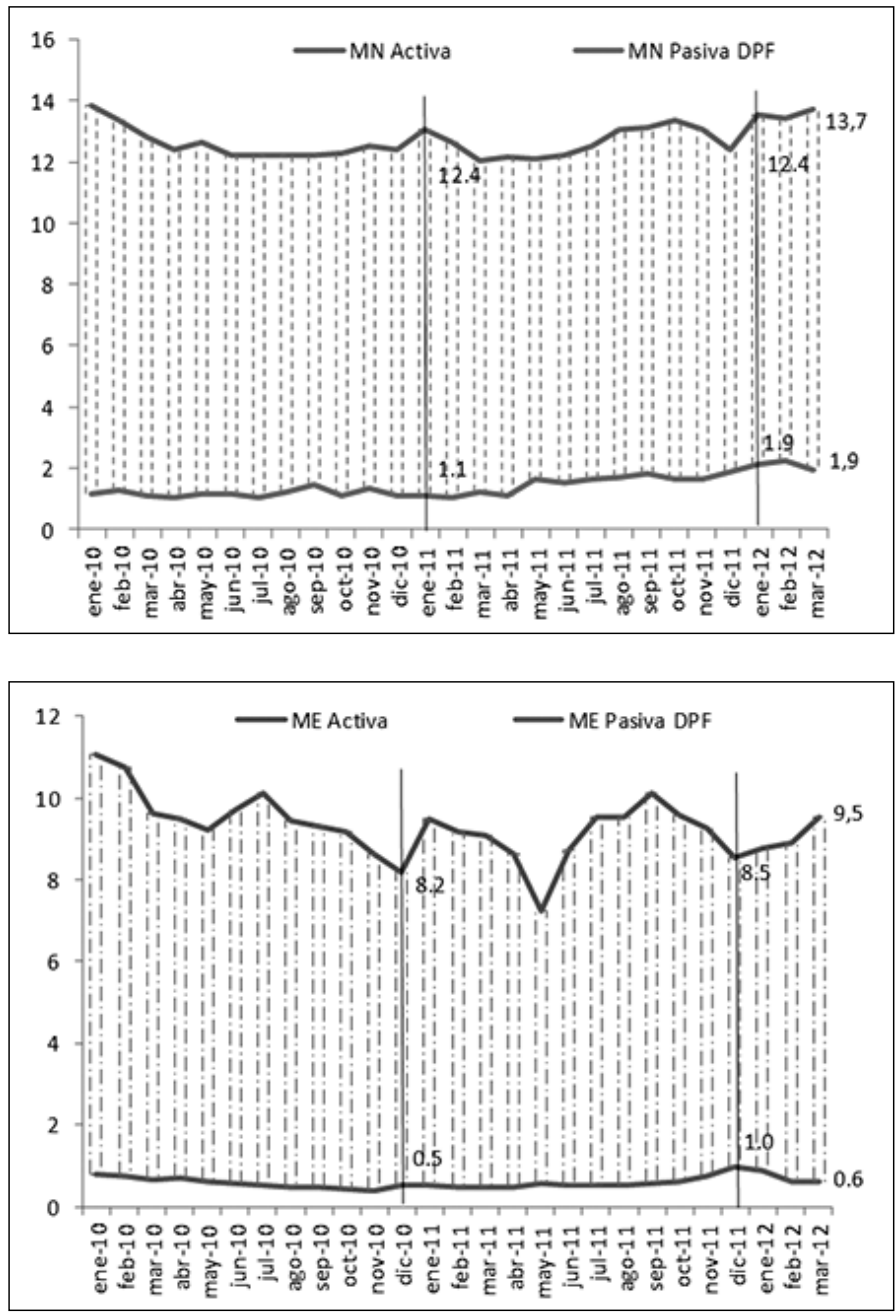

Elaboración propia con datos de la Gerencia de Entidades Financieras del Banco Central de Bolivia. 


\section{Ejercicio en términos de la revalorización de la cartera de inversiones}

Como se vio en el apartado de la metodología de riesgo de tasas de interés, la duración aproxima la elasticidad del precio de un instrumento financiero ante cambios en las tasas de interés. En este sentido, a través de esta definición se determinaron los cambios en el valor del portafolio de inversiones del sistema bancario.

Se estimó la duración de los títulos-valores de la banca a partir de los bonos del Tesoro $(\mathrm{BT})^{19}$, ya que éstos representan el $35 \%$ de las inversiones en entidades públicas no financieras del país que realizan las $\mathrm{EIF}^{20}$, la cual forma parte de la cuenta de inversiones permanentes e inversiones temporarias ${ }^{21}$, ambas a diciembre de 2011. Por lo tanto, se consideró para fines prácticos que estos títulos son los más representativos de la banca.

La duración estimada de las inversiones del sistema bancario $^{22}$, en promedio, fue de 1.97 años, lo cual indica que el plazo promedio de retorno de los títulos mantenidos por los bancos es de 1 año y 11 meses. Con este dato se calculó el impacto de una subida en la tasa de interés en la cartera de inversiones.

\subsection{Riesgo de tipo de cambio ${ }^{23}$}

El riesgo de tipo de cambio se refiere a las pérdidas que podría enfrentar una EIF ante movimientos del tipo de cambio que modifiquen el valor en moneda doméstica de los activos y pasivos contraídos en moneda extranjera.

Este riesgo puede descomponerse en tres efectos:

- El efecto directo a la solvencia, el cual proviene de la revalorización de la posición global neta en moneda extranjera de la banca.

19 Estos son valores a rendimiento, nominativos, emitidos con una tasa de rendimiento nominal preestablecida (tasa cupón), con pagos semestrales de cupones. El precio de venta con respecto a la par determina el rendimiento efectivo. Estos instrumentos se emiten actualmente en MN.

20 Cuenta 164.00, ASFI

21 Cuenta 124.00, ASFI

22 Para calcular la duración es necesario disponer de las condiciones financieras de cada título: precio de corte del ejercicio (en este trabajo es el 31 de diciembre de 2011), el cupón, la fecha de vencimiento (el cálculo necesita los días restantes del vencimiento del título), el monto, la frecuencia del pago de los cupones y la tasa interna de retorno (TIR). Este cálculo para el sistema bancario resultó una tarea ambiciosa por la disponibilidad de la información y la cantidad de títulos. En este sentido, se utilizó la información de los BT del sistema bancario para la fecha de corte del ejercicio.

23 La ASFI aprueba y pone en vigencia el 4 de julio de 2008 el Reglamento de Gestión por Tipo de Cambio, incorporándolo en el Título IX Capítulo XVIII de la Recopilación de Normas de Bancos y Entidades Financieras. 
- El efecto indirecto a la solvencia, que surge cuando se afecta la capacidad de repago de un deudor (cliente del banco), lo que se traduce en un incremento de la morosidad crediticia;

- El riesgo de tipo de cambio referido a la liquidez, la cual vincula las salidas de depósitos con una depreciación del tipo de cambio.

\section{Efecto directo}

Para estimar el riesgo cambiario se utilizó en primera instancia la posición global neta en moneda extranjera al 31 de diciembre de 2011, la cual es calculada mediante el método de Shorthand ${ }^{24}$. Por tanto, el cálculo de la estimación del riesgo por tipo de cambio proviene de la suma de la máxima posición neta global (corta o larga). Para ilustrar esto se seguirá la demostración planteada por Cihak (2007) y Hernández, Días y Valero (2007). Pensemos que $F$ representa la posición neta en moneda extranjera, $C$ el capital, $A_{C R}$ los activos computables por riesgo (todos en moneda nacional), y e la tasa de cambio de la moneda doméstica (boliviano) respecto al dólar. Una depreciación en el tipo de cambio lleva a una revalorización proporcional en la posición neta en moneda nacional $\Delta e / e \cong \Delta F / F$ para $F \neq 0$. Ahora asumimos que este efecto se transmite directamente al capital $\Delta C / \Delta F=1$. Por lo tanto, el impacto de una devaluación del tipo de cambio en el CAP será:

$$
\frac{\Delta\left[C(e) / A_{C R}(e)\right]}{\Delta e} \cong \frac{\frac{F}{e} A_{C R}-C \frac{\Delta A_{C R}}{\Delta C} \frac{F}{e}}{A_{C R}} \cong \frac{1}{e} \frac{F}{C} \frac{C}{A_{C R}}\left(1-\frac{\Delta A_{C R}}{\Delta C} \frac{C}{A_{C R}}\right)
$$

Reescribiendo la ecuación (9), siendo que $\Delta C / \Delta e \cong \Delta F / \Delta e \cong F / e$, tenemos:

$$
\Delta\left[C(e) / A_{C R}(e)\right] \cong \frac{\Delta e}{e} \frac{F}{C} \frac{C}{A_{C R}}\left(1-\frac{\Delta A_{C R}}{\Delta C} \frac{C}{A_{C R}}\right)
$$

El término $\Delta A_{C R} / \Delta C$ oscila entre 1 y 0 reflejando los movimientos conjuntos de estas dos variables, a saber, la variación del CAP se calcularía como $\frac{\Delta e}{e} \frac{F}{C} \frac{C}{A_{C R}}$, lo que representaría un método rápido y valioso para aproximar el impacto directo de una depreciación. Según Cihak (2007), esta aproximación tiene un buen desempeño en sistemas financieros no sofisticados ${ }^{25}$

24 Este método asigna un tratamiento igualitario a todas las monedas extranjeras, bajo el supuesto de que el efecto de las fluctuaciones entre monedas es proporcional

25 En sistemas financieros sofisticados, donde las entidades financieras tienen opciones de monedas, la relación de transmisión lineal al CAP anteriormente presentado se vuelve altamente no lineal, por lo tanto, es necesario 
como el caso de Bolivia, en otras palabras, las instituciones financieras en Bolivia no poseen instrumentos derivados como opciones de monedas.

\section{Efecto indirecto}

Este efecto se refiere al cambio en la cartera en mora ante una apreciación o depreciación del tipo de cambio. Conceptualmente, una variación del tipo de cambio podría afectar a las firmas en dos aspectos: primero, alterando la competitividad externa de este sector; $y$ segundo, revalorizando las cuentas del balance de la compañía. A su vez, este segundo aspecto tendría un efecto en el balance de los bancos, dependiendo su posición de moneda neta. Al no existir información precisa sobre el grado de exposición de las firmas, se desarrolló este ejercicio mediante supuestos de comportamiento.

De acuerdo al modelo estimado en el apartado de riesgo crediticio, sí opera el efecto negativo de una depreciación del tipo de cambio sobre la cartera en mora. Esto es razonable dado que las depreciaciones están ligadas a periodos de alta inflación en Bolivia (ejemplo: 1956, 1985), los cuales a su vez afectaron al sistema financiero. El efecto indirecto de una devaluación de tipo de cambio sobre la liquidez se lo presentará en el siguiente apartado.

\subsection{Riesgo de liquidez ${ }^{26}$}

Para este ejercicio se plantearon dos escenarios. El primero se refiere a un retiro proporcional para todos los bancos donde, siguiendo al Informe de Estabilidad Financiera (Julio de 2012) del Banco Central de Bolivia, se replicó el comportamiento de la máxima salida de depósitos, ocurrida entre el 4 de julio y el 5 de agosto de 2002, cuando, en promedio, se registró una disminución del 14\% del total de los depósitos del sistema financiero. El segundo se basa en retiros diferenciados en función del tamaño del activo de las entidades bancarias. A continuación se presenta el modelo VEC de liquidez, el cual será utilizado para el ejercicio referido a los retiros proporcionales de depósitos.

realizar otro tipo de análisis.

26 El riesgo de liquidez se interpreta como el número de días que una institución financiera puede sobrevivir ante una situación de retiros masivos de depósitos; todo ello sin contar con la asistencia crediticia de las ventanillas de liquidez del Banco Central. 


\section{Modelo VEC de liquidez}

Para realizar el ejercicio de ST se estimó una relación de largo plazo entre las variables presentadas a continuación, a partir de un modelo de corrección de errores (VEC) con periodicidad trimestral, que vincula las obligaciones públicas de las EIF como variable endógena, con variables exógenas escogidas por investigaciones similares (Hernández et al., 2007; Delgado, 2004).

- Logdepo: es el logaritmo de las obligaciones con el público del sistema bancario.

- Dev: es la primera diferencia del tipo de cambio nominal en logaritmo.

- Logpibcons_sa: es el PIB de construcción en logaritmos suavizada por el método Census X-12 dado su comportamiento estacional (se reduce) en el cuarto trimestre.

- T_pasiva: es la tasa promedio de interés pasiva efectiva en moneda extranjera del sistema bancario boliviano.

- logipc: es el índice de precios al consumidor con año base 2007

Como se aprecia en el Cuadro 3, las variables resultaron de orden de integración I(1). Los resultados de la prueba de cointegración de Johansen mostraron que existe al menos una combinación lineal entre estas variables, que da un vector estacionario (véase Anexo 1). La regresión no presentó problemas de heteroscedasticidad ni autocorrelación.

Cuadro 3

Contraste de raíces unitarias

\begin{tabular}{ccccccc}
\hline \multirow{3}{*}{ Variables } & \multicolumn{3}{c}{ Niveles } & \multicolumn{3}{c}{ Primeras diferencias } \\
\cline { 2 - 7 } & $\begin{array}{c}\text { A. Dickey-Fuller } \\
\text { P-Valor }\end{array}$ & Rezagos & $\begin{array}{c}\text { Pillips-Perron } \\
\text { P-Valor }\end{array}$ & $\begin{array}{c}\text { A. Dickey-Fuller } \\
\text { P-Valor }\end{array}$ & $\begin{array}{c}\text { Phillips-Pezagos } \\
\text { P-Valor }\end{array}$ \\
\hline logdepo & 0.9999 & 1 & 0.9996 & 0.0041 & 0 & 0.0077 \\
dev & 0.2568 & 1 & 0.2324 & 0.0000 & 0 & 0.0000 \\
logpibcons_sa & 0.5390 & 0 & 0.7329 & 0.0000 & 1 & 0.0001 \\
logtasa_pas & 0.5381 & 1 & 0.8027 & 0.0000 & 0 & 0.0000 \\
logipc & 0.5450 & 1 & 0.5140 & 0.0000 & 0 & 0.0000 \\
\hline
\end{tabular}

Notas: Los p-valores son de una cola según MacKinnon. Los rezagos del test ADF se eligieron de acuerdo al criterio de Schwarz y viendo que las regresiones auxiliares sean por lo menos ruido blanco.

A priori se esperaría un incremento en el IPC o en la tasa de devaluación se traduciría en una disminución de los depósitos, dado el costo de oportunidad que implicaría la tenencia de 
estos últimos en moneda local, con lo cual se espera que los signos de los coeficientes sean negativos.

En el caso de la depreciación, podría ser que las personas cambien sus depósitos en MN por ME. Sin embargo, el componente de histéresis inflacionaria a través del tipo de cambio puede provocar una salida de depósitos inesperada en MN.

Por otra parte, el signo esperado del coeficiente que acompaña a la tasa de interés pasiva es positivo, existirá mayor incentivo de mantener activos remunerados en la medida que se produzcan incrementos de las tasas. Por último, se halló evidencia de que la tendencia de los depósitos mantiene una relación de largo plazo más robusta con el PIB de la construcción que con el PIB total. En este sentido, al ser una medida de ingreso, se esperaría que tenga un signo positivo, ya que al incrementarse el ingreso se produciría un efecto positivo en los depósitos.

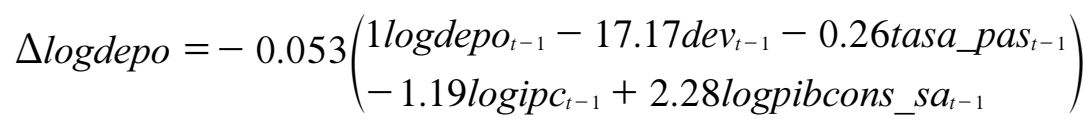

De acuerdo al resultado del vector de largo plazo ${ }^{27}(11)$, un incremento en la tasa de devaluación se traduciría en una disminución de los depósitos, dado el costo de oportunidad que implicaría la tenencia de estos últimos en moneda local; asimismo, un incremento del IPC produciría una disminución en los depósitos, dado el costo de oportunidad que implicaría mantener los depósitos en el banco. Sin embargo, el signo del coeficiente que acompaña a la tasa de interés pasiva es negativo. Esta incoherencia con la teoría se debe al exceso de liquidez existente en la economía boliviana, y a un fenómeno de demanda: el primero se refiere al exceso de material monetario en la economía, que se plasma en incrementos significativos de los depósitos en los últimos seis años, esto a pesar que la tasa pasiva sigue disminuyendo; el segundo se refiere a la movilidad social que se ha dado en los últimos años y la confianza en el sistema financiero, que ha posibilitado que se incremente el número de depositantes en el sistema financiero.

Por último, es importante notar que el porcentaje de retiros ante una devaluación marginal es similar a las salidas de depósitos entre julio y agosto de 2012 (14\%). Como discutimos en la parte de elaboración de un ejercicio de ST, lo más importante en este tipo de ejercicios

27 Las variables del modelo resultaron ser significativas al 10\%, y los residuos no presentan problemas de autocorrelación ni heteroscedasticidad (véase Anexo 1). 
es la plausibilidad de los escenarios, es decir, tanto los escenarios como las sensibilidades tienen que ser lo más reales posibles. De esta manera se procedió a utilizar la elasticidad ${ }^{28} \mathrm{de}$ la devaluación sobre los depósitos (17.17\%) para el ejercicio de retiros proporcionales de liquidez en el módulo de Riesgo de Liquidez. Como bien dijimos en la estructura del ST, la variación del tipo de cambio implica también retiros de depósitos.

\section{Resultados}

\subsection{Análisis de riesgos por módulos}

En la sección anterior se describieron los fundamentos teóricos y la metodología a ser utilizada. En este apartado se exponen los resultados por módulo (riesgo crediticio, riesgo cambiario, riesgo de tasa de interés y riesgo de liquidez), correspondientes a la aplicación de los cuatro escenarios macroeconómicos seleccionados. Una vez analizados éstos, se expondrán los resultados de forma integrada.

\section{Riesgo de crédito}

Los escenarios planteados implican básicamente subidas de las tasas de interés, excepto el escenario de crisis asiática, que muestra una depreciación del 4\% (véase Cuadro 4). Cuando esto ocurre, se incrementa la cartera en mora, siendo necesario el incremento en provisiones netas (descontándole la tasa de colateral), las cuales reducen el activo ponderado por riesgo y el patrimonio neto, llevando a una disminución en el CAP del banco. Siguiendo el Informe de Estabilidad Financiera (julio de 2012) del Banco Central de Bolivia, se asumió que los créditos están colateralizados y que, cuando ocurre un deterioro de la cartera en mora, la tasa de recuperación del colateral es del $80 \%^{29}$, y las nuevas provisiones representan $100 \%$ de los nuevos préstamos vencidos y en ejecución, previo descuento de la tasa de recuperación del colateral (Cuadro 5).

Los resultados de los escenarios con relación al impacto en el CAP del sistema financiero mostraron resultados moderados, excepto el escenario de crisis asiática, el cual resulto el más adverso, con una caída de 0.48 puntos porcentuales del CAP. Sin embargo, en ningún

28 Este parámetro de retiro de depósitos difiere en 3.17\% con relación al registrado durante la salida de depósitos ocurrida entre el 4 de julio y el 5 de agosto de 2002, cuando, en promedio, salieron del sistema financiero el $14 \%$ del total de depósitos.

29 En el Anexo 4 se muestra el mismo ejercicio, con una tasa de colateral del $25 \%$. Este coeficiente es empleado por Cihak (2007) y Hernández et al. (2007). 
escenario el CAP se situó por debajo del nivel establecido por el regulador $(10 \%)$ o por Basilea (8\%). En este sentido, se concluye que el riesgo de crédito no parece constituir una de las mayores vulnerabilidades del sistema financiero, es decir, a partir de los escenarios planteados, las variaciones en el CAP resultaron ser marginales, lo cual es razonable, dado el nivel de pesadez que tiene el sistema bancario boliviano a 2011 (1.65\%), el mínimo valor registrado en la historia desde 1999 (véase Gráfico 5). Asimismo, el hecho de que los bancos tengan un alto porcentaje de cartera resguardada por un colateral significa que el aumento requerido de provisiones no causa un efecto significativo en el CAP del sistema bancario.

\section{Gráfico 5: Evolución de la pesadez en el sistema bancario (en porcentajes)}

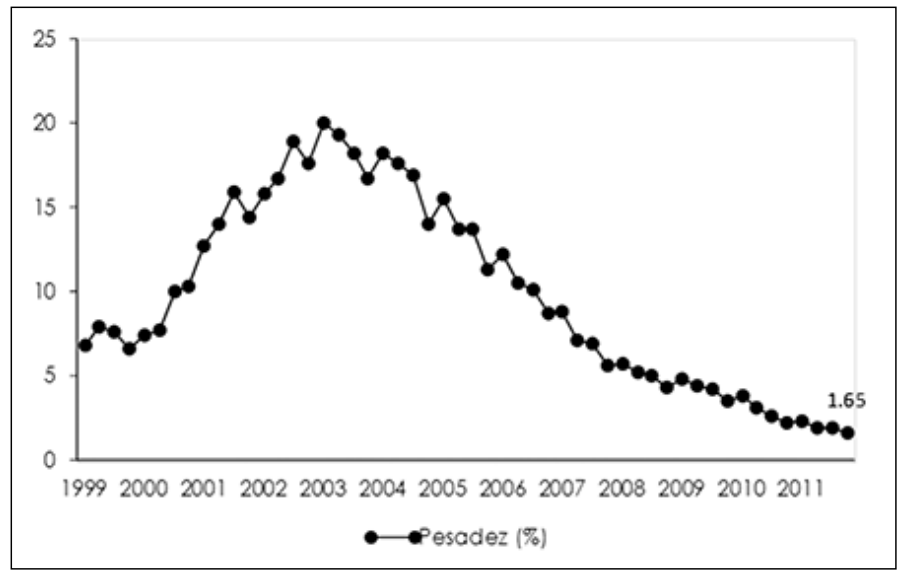

Elaboración propia con datos de los boletines de bancos de la ASFI 


\section{Cuadro 4}

Riesgo de crédito (en millones de bolivianos y porcentajes)

\begin{tabular}{|c|c|c|c|c|}
\hline Efecto provisiones & $\begin{array}{l}\text { Crisis } \\
\text { asíatica }\end{array}$ & $\begin{array}{l}\text { Inestabilidad } \\
\text { política }\end{array}$ & $\begin{array}{l}\text { Shock de } \\
\text { créditos }\end{array}$ & $\begin{array}{l}\text { Subida del } \\
\text { encaje legal }\end{array}$ \\
\hline Pesadez (\%) & 1.65 & 1.65 & 1.65 & 1.65 \\
\hline Increm ento en la cartera en mora (\%) & 39.68 & 24.22 & 21.34 & 15.00 \\
\hline $\begin{array}{l}\text { Nueva cartera en mora (Estimado vs } \\
\text { observadol }\end{array}$ & 301.25 & 161.06 & 162.00 & 113.90 \\
\hline Nuevos préstamos vencidos & 150.63 & 80.53 & 81.00 & 56.95 \\
\hline Nuevos préstamos en ejecución & 150.63 & 80.53 & 81.00 & 56.95 \\
\hline Pesadez poschoque (\%) & 2.31 & 2.00 & 2.01 & 1.90 \\
\hline $\begin{array}{l}\text { Cobertura del colateral de los } \\
\text { nuevos créditos morosos }(\%)\end{array}$ & 80 & 80 & 80 & 80 \\
\hline $\begin{array}{l}\text { Nuevas provisiones no cubiertas por } \\
\text { el colateral (+ Increm ento/- } \\
\text { reducción) }\end{array}$ & 60.25 & 32.21 & 32.40 & 22.78 \\
\hline Nuevos préstam os vencidos (100\%) & 30.13 & 16.11 & 16.20 & 11.39 \\
\hline $\begin{array}{l}\text { Nuevos préstam os en ejecución (100\%) } \\
\text { Impacto total en las nuevas provisiones (+ }\end{array}$ & 30.13 & 16.11 & 16.20 & 11.39 \\
\hline $\begin{array}{l}\text { mayor capitalización/ - menor } \\
\text { capitalización ) }\end{array}$ & -60.25 & -32.21 & -32.40 & -22.78 \\
\hline $\begin{array}{l}\text { Coeficiente de Adecuación Patrimonial } \\
\text { (CAP) inicial (\%) }\end{array}$ & 12.27 & 12.27 & 12.27 & 12.27 \\
\hline Capital computable poschoque & 5728.64 & 5752.12 & 5756.49 & 5766.11 \\
\hline Activo computable poschoque & 47117.71 & 47141.18 & 47145.55 & 47155.18 \\
\hline $\begin{array}{l}\text { Coeficiente de Adecuación Patrimonial } \\
\text { (CAP) poschoque provisiones (\%) }\end{array}$ & 12.16 & 12.20 & 12.21 & 12.23 \\
\hline Cambio en el CAP (pp) & -0.11 & -0.07 & -0.06 & -0.042 \\
\hline $\begin{array}{l}\text { Capital requerido según el 10\% de CAP } \\
\text { requerido por la ASFI }\end{array}$ & 4717.79 & 4717.79 & 4717.79 & 4717.79 \\
\hline $\begin{array}{l}\text { Excedente (déficit) patrimonial de la } \\
\text { banca (milles de bs.) }\end{array}$ & 1010.85 & 1034.32 & 1038.69 & 1048.32 \\
\hline $\begin{array}{l}\text { Excedente (déficit) patrimonial de la } \\
\text { banca (\% PIB) }\end{array}$ & 0.73 & 0.75 & 0.75 & 0.76 \\
\hline
\end{tabular}

\section{Riesgo de tasas de interés}

Este ejercicio tiene dos partes. La primera se refiere al flujo de intereses neto proveniente de los ingresos recibidos por los créditos y los egresos por la tenencia de los depósitos. La segunda evalúa el impacto de la revalorización del portafolio de inversiones de las instituciones bancarias. Para la primera parte se calculó el GAP o brecha individual acumulada a partir de los plazos de vencimiento de la cartera (créditos) y los depósitos (obligaciones con el público). Cabe destacar que la brecha acumulada es negativa, llegando a Bs. 4.470 millones. Este descalce entre los plazos a los que se colocan créditos y a los que se captan fondos podría 
incrementar la vulnerabilidad del sector bancario ante un choque en las tasas de interés o de liquidez (corrida de depósitos). Para la segunda parte, la duración estimada de las inversiones del sistema bancario, en promedio, fue de 1.97 años, lo cual indica que el plazo promedio de retorno de los títulos mantenidos por los bancos son de 1 año y 11 meses. Con este dato se calculó el impacto de una subida en la tasa de interés en la cartera de inversiones.

Los resultados muestran que los aumentos en la tasas de interés, manteniendo el spread constante, producen disminuciones significativas de los escenarios de crisis asiática, inestabilidad política, shock de créditos y subida del encaje legal en el CAP de 0.4 puntos porcentuales (pp), 1.95pp, 2.04pp, y 1.43pp, respectivamente. Los escenarios que tuvieron mayor impacto en el CAP (2.04 pp y 1.95pp) fueron los correspondientes al escenario de inestabilidad política y al shock de créditos. Sin embargo, en ninguno de los casos el CAP cae por debajo del nivel establecido por el regulador (10\%).

A partir de los resultados, se intuye que el riesgo de tasas de interés tiene un impacto importante en el CAP de los bancos dado su descalce de plazos, lo cual muestra un mayor grado de vulnerabilidad o de exposición del sistema bancario ante modificaciones en la tasa de interés (véase Cuadro 5).

\section{Riesgo de tipo de cambio}

Este ejercicio consta de dos partes. La primera se refiere al efecto directo que opera en función de la posición cambiaria en moneda extranjera ${ }^{30}$. La segunda considera el efecto indirecto que pueden tener variaciones en el tipo de cambio en la cartera en mora.

Para el primer caso, a partir de la posición cambiaria en moneda extranjera (en dólares) se evaluaron los cuatro escenarios macroeconómicos en los que se introducen choques cambiarios de diferentes magnitudes y direcciones, para obtener el impacto en el CAP. Dado el tipo de cambio que opera en Bolivia (Crowling peg) a partir de 1985, no es posible encontrar un escenario adverso en términos de variación de éste, más que el que corresponde a la crisis asiática. En este sentido, para fines ilustrativos se introdujeron dos escenarios hipotéticos para mostrar el impacto de una depreciación cambiaria en la cartera en mora: Depreciación súbita I y II (véase Cuadro 6). Estos escenarios contemplan una depreciación de 20pp y 40pp, respectivamente. Por otra parte, dada la posición neta larga en moneda extranjera que posee el sistema bancario boliviano, una depreciación del tipo de cambio impacta positivamente

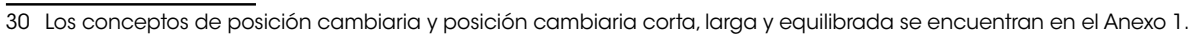


en los balances, mientras que una apreciación tiene un efecto desfavorable en los mismos. Es decir, una depreciación del tipo de cambio contribuiría a la capitalización de la banca. Esto debido a que un aumento del tipo de cambio favorece a aquellos bancos que contienen una posición larga (positiva) en moneda extranjera, y como en general el sistema bancario boliviano se encuentra en este contexto, una depreciación podría coadyuvar a un aumento en su patrimonio. El segundo caso se refiere al apartado de riesgo de crédito, el cual muestra que una depreciación del tipo de cambio implicaría un incremento en la cartera en mora. Esto a partir del supuesto de que la memoria inflacionaria afecta a las decisiones de corto y largo plazo de los agentes, que toman en cuenta en sus decisiones la historia inflacionaria y la incertidumbre (Sheriff, 2010).

Los resultados presentados en el Cuadro 7 muestran que en los escenarios históricos seleccionados el efecto del riesgo cambiario es bajo, dado que no muestran variaciones del tipo de cambio precipitadas. En cambio, sí opera el efecto del riesgo cambiario en los escenarios hipotéticos; sin embargo, éstos muestran no ser totalmente adversos, dada la posición larga en ME que cobertura el posible efecto indirecto anteriormente mencionado. 


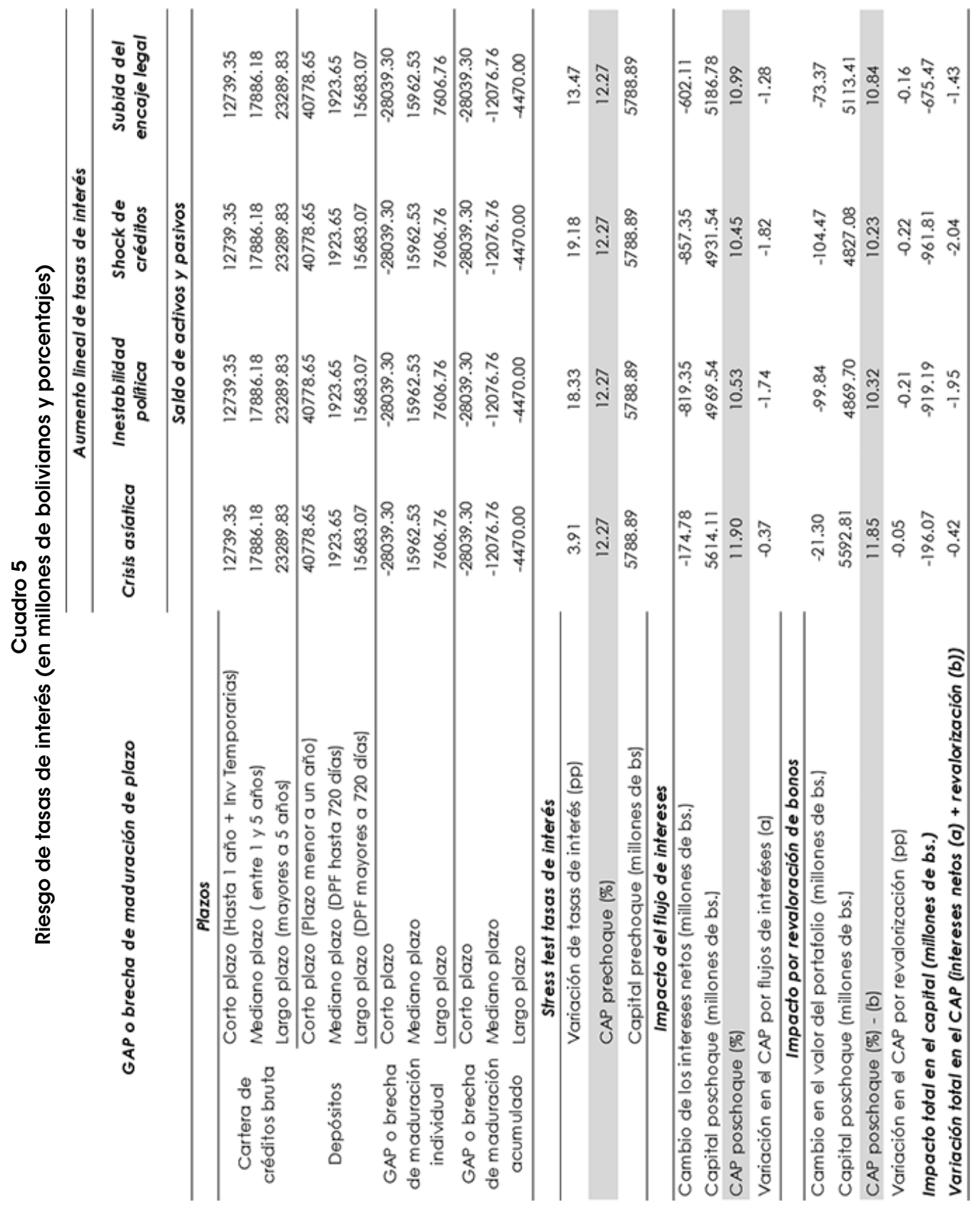




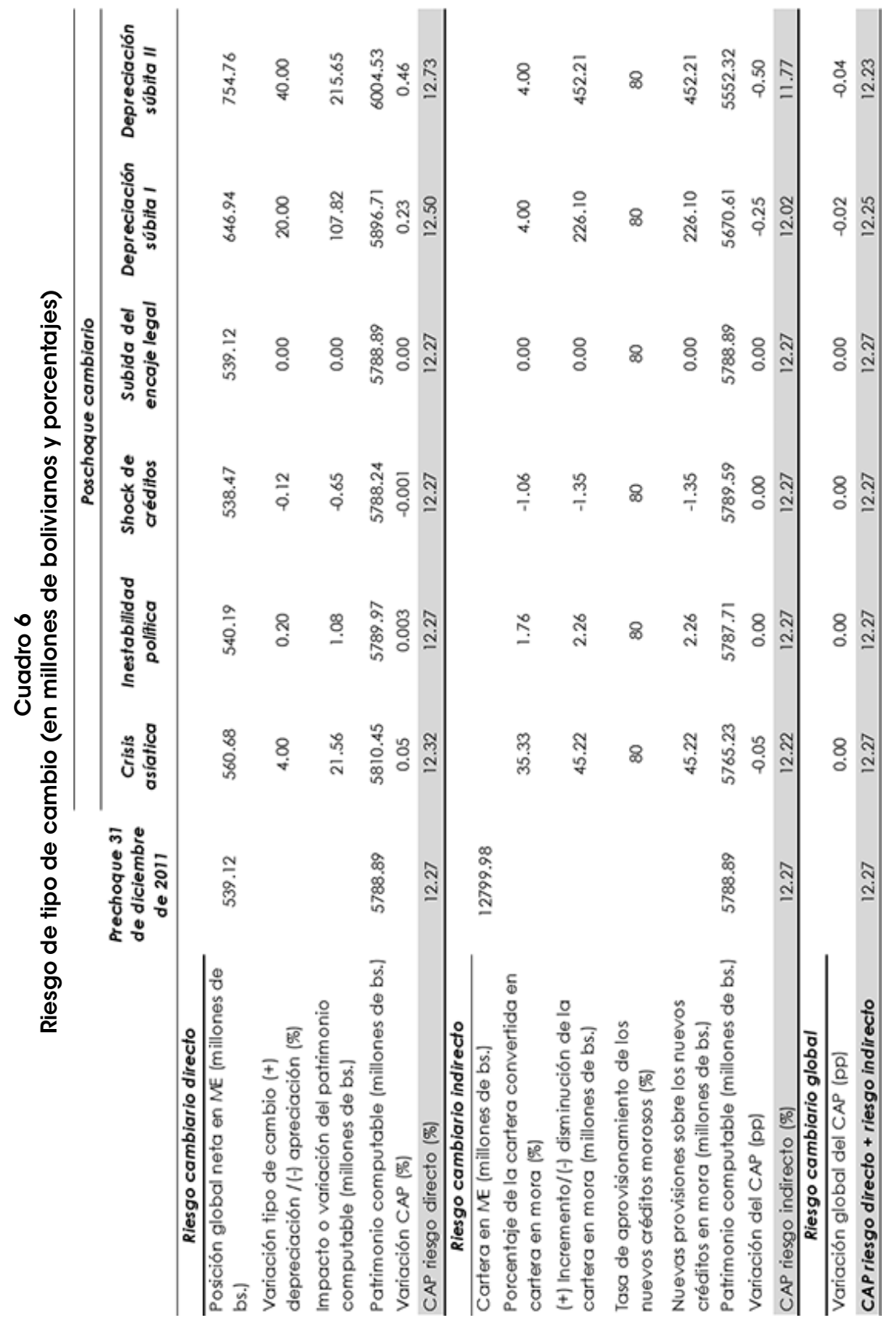




\section{Riesgo de liquidez}

\section{Variables utilizadas $^{31}$}

Para realizar el ejercicio, los depósitos fueron divididos en dos grupos en función a su grado de convertibilidad en efectivo: el primer grupo, el más líquido, está conformado por depósitos vista y ahorro en distintas denominaciones. El segundo grupo considera únicamente los depósitos a plazo hasta 30 días. Por su parte, las cuentas del activo fueron desglosadas en activos líquidos e ilíquidos, de acuerdo a la rapidez con la cual se convierten en efectivo. Los activos líquidos incluyen las siguientes cuentas: disponibilidades (efectivo, encaje legal) e inversiones en temporarias que se refieren a títulos valores convertibles en efectivo en un plazo menor a 90 días. Los activos ilíquidos se calculan por diferencia.

\section{Retiros proporcionales de depósitos}

El propósito del ejercicio es determinar, durante cinco días consecutivos, el grado de liquidez de cada banco que se enfrenta a una salida masiva de depósitos. Es decir, en el ejercicio se simulan por cinco días consecutivos retiros diarios equivalentes a un porcentaje determinado con base en la elasticidad de la devaluación sobre los depósitos (17.17\%), proveniente del modelo de corrección de errores (VEC) de depósitos expuesto en el módulo de riesgo de liquidez. Estas salidas estarían cubiertas con los activos líquidos (100\%) y con un $1 \%$ de los activos ilíquidos. Diariamente se calcula un indicador de liquidez para cada banco, el cual toma valor 1 si la liquidez disponible supera los retiros, y 0 en caso contrario. De esta manera es posible determinar cuáles bancos soportan los retiros de depósitos y cuáles no.

Los resultados se presentan en el Cuadro 6, donde se observa que ninguno de los bancos tiene problemas de liquidez al cabo de 5 días. Cabe destacar que no se toma en cuenta el hecho de que en caso de requerirlo, los bancos pueden acceder al mercado interbancario o a las ventanillas de liquidez del Banco Central de Bolivia. En este sentido, se podría vincular estos buenos resultados con el desempeño favorable de banca en los últimos años y la buena gestión de liquidez de los bancos a nivel micro, así como al hecho de que en los últimos años se registraron niveles altos de liquidez en el sistema financiero boliviano.

31 Estas variables se encuentran en los boletines de bancos de la Autoridad de Supervisión del Sistema Financiero (ASFI). 


\section{Gráfico 6: Liquidez disponible I (en miles de bolivianos) ${ }^{32}$}

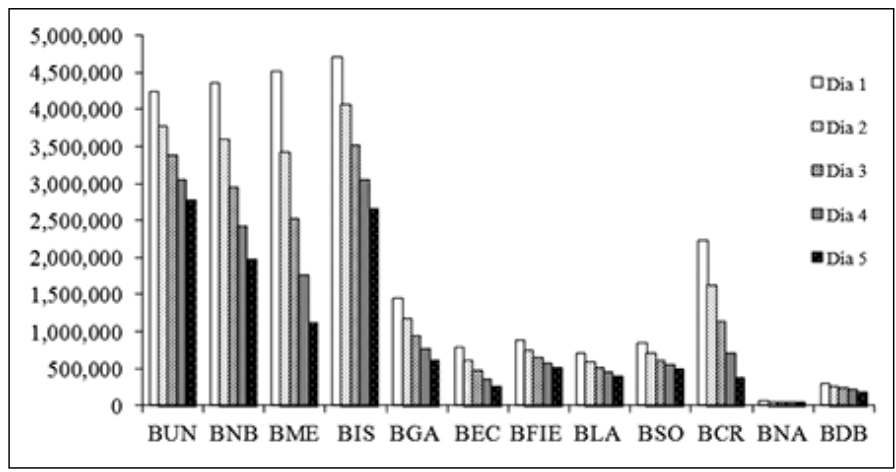

Fuente: Elaboración propia con datos de los boletines de bancos de la ASFI

\section{Retiros diferenciados de depósitos}

A diferencia del apartado anterior, en este ejercicio se propone una salida de retiros diferenciados con relación al tamaño de cada banco, es decir, en función a su activo total. Se calculó un indicador que va de 1 (el banco con mayor total activo) a 0 (el banco con menor total activo), para determinar el porcentaje de retiro de depósitos diarios. A su vez este porcentaje varía desde un $01 \%$ a $25 \%$ con relación al indicador. De esta manera, se intuye que los bancos más grandes sufrirán mayores salidas de depósitos con relación a los bancos pequeños.

Los resultados muestran que ninguno de los bancos tiene problemas de liquidez al cabo de 5 días; sin embargo, el Banco Mercantil se encuentra muy cercano a fallar la prueba (véase Gráfico 7). Es preciso destacar que el impacto (25\%) no es significativamente distinto al porcentaje de salidas del Banco Nacional de Bolivia (21\%), el cual se posiciona en el segundo lugar por su activo total. Por lo tanto, su nivel de liquidez no es óptimo con relación a su tamaño. Por otra parte, los demás bancos no muestran cambios significativos en términos de su capacidad de cerrar posiciones con el público, con relación al apartado anterior (véase Gráfico 7).

32 "Como regla general, algunos supervisores analizan 5 días como un umbral importante para la capacidad de un banco para soportar una liquidez de ejecución. El número 5 es típicamente elegido porque después de 5 días o menos, los bancos cerrarán durante un fin de semana o un día de fiesta, dando un poco de "tiempo de respiro" para la gestión bancaria y supervisores para reagruparse, evaluar la situación y decidir sobre las medidas y anuncios públicos por hacer. Por supuesto, esta regla tradicional de pulgar ha sido parcialmente diluida con el crecimiento de la relación directa que mantiene el banco con su cliente (y en particular con la Internet)" (Cihak, 2007). 
Gráfico 7: Liquidez disponible II (en miles de bolivianos)

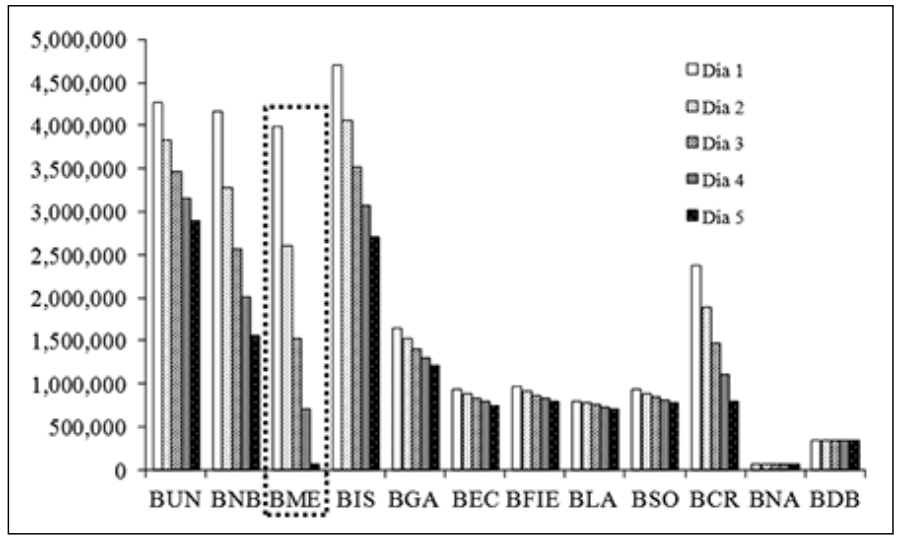

Fuente: Elaboración propia con datos de los boletines de bancos de la ASFI

\subsection{Análisis de riesgos agregados}

En el Gráfico 8 se presentan los resultados de los movimientos de los factores de riesgo (tasas de interés y tipo de cambio) de los cuatro escenarios planteados, con sus respectivos valores agregados de CAP poschoque, comparados con el CAP inicial (12.27\%), correspondiente a diciembre de 2011. Para cada escenario se sumaron las variaciones del CAP producidas por cada riesgo (salvo el de liquidez). De los cuatro escenarios planteados, los de mayor impacto sobre la banca son los correspondientes al shock de créditos e inestabilidad política, con variaciones negativas en el CAP de $2.10 \%$ y $2.01 \%$, respectivamente (véase Cuadro 7 ). Sin embargo, en ninguno de los escenarios considerados el CAP del sistema bancario cae por debajo del 10\% establecido por la ASFI ni por Basilea (8\%), lo cual muestra que el sistema bancario, como se menciona en la memoria institucional de la ASFI a 2011, se encuentra sólido a 2011. Por otra parte, es preciso enfatizar que los escenarios adversos corresponden a subidas de la tasa de interés, y dada la vulnerabilidad detectada del sistema bancario boliviano ante cambios en las tasas de interés, la diferencia entre los dos escenarios más adversos fue determinada por la variación más alta de tasas de interés, la cual implica el efecto más adverso en el CAP. 


\section{Gráfico 8: Cambio global en el CAP según escenario (en porcentajes)}

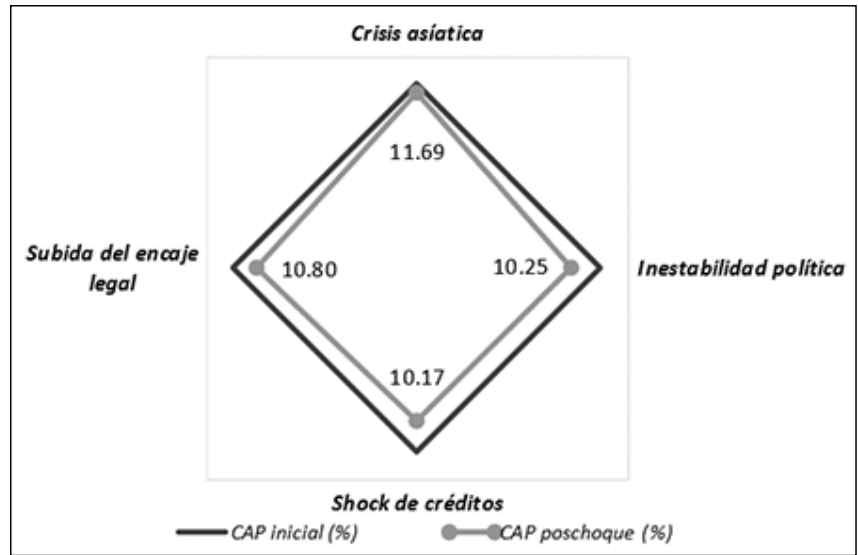

Nota: Puntos más cercanos del origen implican una disminución del CAP con respecto al CAP inicial (12.27\%)

Fuente: Elaboración propia

\section{Cuadro 7 \\ Resumen de los resultados (en porcentajes)}

\begin{tabular}{lccccc}
\cline { 2 - 6 } \multicolumn{1}{c}{ Escenarios } & CAP inicial (\%) & $\begin{array}{c}\text { CAP poschoque } \\
\text { (\%) }\end{array}$ & $\begin{array}{c}\text { Variación del } \\
\text { CAP (Pp) }\end{array}$ & $\begin{array}{c}\text { Variación del } \\
\text { tipo de cambio } \\
(\mathbf{P p})\end{array}$ & $\begin{array}{c}\text { Variación de la } \\
\text { tasa de interés } \\
\text { (Pp) }\end{array}$ \\
\hline Crisis asíatica & 12.27 & 11.69 & -0.58 & 4.00 & 3.91 \\
Inestabilidad politica & 12.27 & 10.26 & -2.01 & 0.20 & 18.33 \\
Shock de créditos & 12.27 & 10.17 & -2.10 & -0.12 & 19.18 \\
Subida del encaje legal & 12.27 & 10.80 & -1.47 & 0.00 & 13.47 \\
\hline
\end{tabular}

Fuente: Elaboración propia

Para analizar los resultados de forma agregada y por grupos de bancos, se vio conveniente un análisis diferenciado según la naturaleza de nacionalidad del banco. Siguiendo la conceptualización de banca extranjera de Hurtado, Villacorta y Ferruz (2008) ${ }^{33}$, se planteó una evaluación de los resultados agregados en función a la nacionalidad del paquete accionario

33 A pesar de que La Ley de Bancos y Entidades Financieras $N^{\circ} 1488$ establece dos modalidades bajo las cuales pueden operar las entidades financieras de propiedad extranjera en el país: Oficina de Representación y Sucursal. La primera es una oficina promotora de negocios que representa a una entidad de intermediación financiera constituida y radicada en el exterior del país; la segunda, en cambio, es una oficina autorizada a operar en el país perteneciente a un banco constituido y radicado en el exterior y sometida a la autoridad administrativa y dependencia organizacional de su casa matriz. Sin embargo, es preciso reconocer una tercera categoría, las llamadas subsidiarias. Una subsidiaria vendría a ser un banco constituido en el país como sociedad anónima, y cuya propiedad pertenece mayoritariamente a una o varias empresas extranjeras relacionadas con la actividad financiera. Esa relación de propiedad supondría, además del control de la casa matriz en la gestión estratégica y operativa de la subsidiaria, transferencias de tecnología, de conocimiento y otros aspectos. 
mayoritario en el banco. En el Cuadro 8 se presenta el número de bancos en cada categoría y su participación en el sistema.

\section{Cuadro 8 \\ Estructura del activo del sistema bancario según nacionalidad del paquete accionario mayoritario}

\begin{tabular}{lcc} 
& Activos & $\boldsymbol{N}^{\circ}$ de Bancos \\
\hline Públicos & 11 & 1 \\
Privados nacionales & 59 & 5 \\
Extranjeros & 30 & 6 \\
\hline \multicolumn{1}{c}{ Total } & 100 & 12 \\
\hline
\end{tabular}

Fuente: Elaboración propia en base al Anuario Estadístico de la ASFI 2011

Tomando en consideración esta clasificación, se calculó el CAP prechoque y poschoque por grupo de bancos para cada uno de los cuatro escenarios macroeconómicos adversos seleccionados. En el escenario relativo a la crisis asiática (véase Cuadro 9), se pudo apreciar que el Banco Unión fue el más afectado ante esta situación, con una variación negativa de $1.87 \mathrm{pp}$, seguido por los bancos privados nacionales (caída del CAP de $0.88 \mathrm{pp}$ ). Cabe destacar que el riesgo de tasas de interés afecta a todo el sistema, y resultó el más importante de todos los riesgos calculados, una vez impactadas las variables macroeconómicas. Esto debido al descalce de plazos calculados. En consecuencia, de la disminución total del capital que experimenta el sistema una vez introducidos los choques, el $72.0 \%$ corresponde al riesgo de tasas de interés, seguido por el riesgo de crédito, con un $19.4 \%$, y, por último, el riesgo cambiario, con $8.6 \%$.

Los escenarios correspondientes a inestabilidad política, shock de créditos y subida del encaje legal se muestran en el Anexo 2. En general, los resultados muestran que los bancos públicos (Banco Unión) y los bancos privados experimentan una variación negativa del CAP ante estos escenarios, mientras que los bancos extranjeros se capitalizan. Esto debido a que los bancos extranjeros tienen un GAP positivo, es decir, hay una relación entre los plazos a los que prestan y a los que captan que les permiten estar resguardados del riesgo de tasas de interés. Bajo este esquema, una variación de las tasas de interés los capitaliza. 


\section{Cuadro 9}

Escenario: Crisis asiática (en millones de bolivianos y porcentajes)

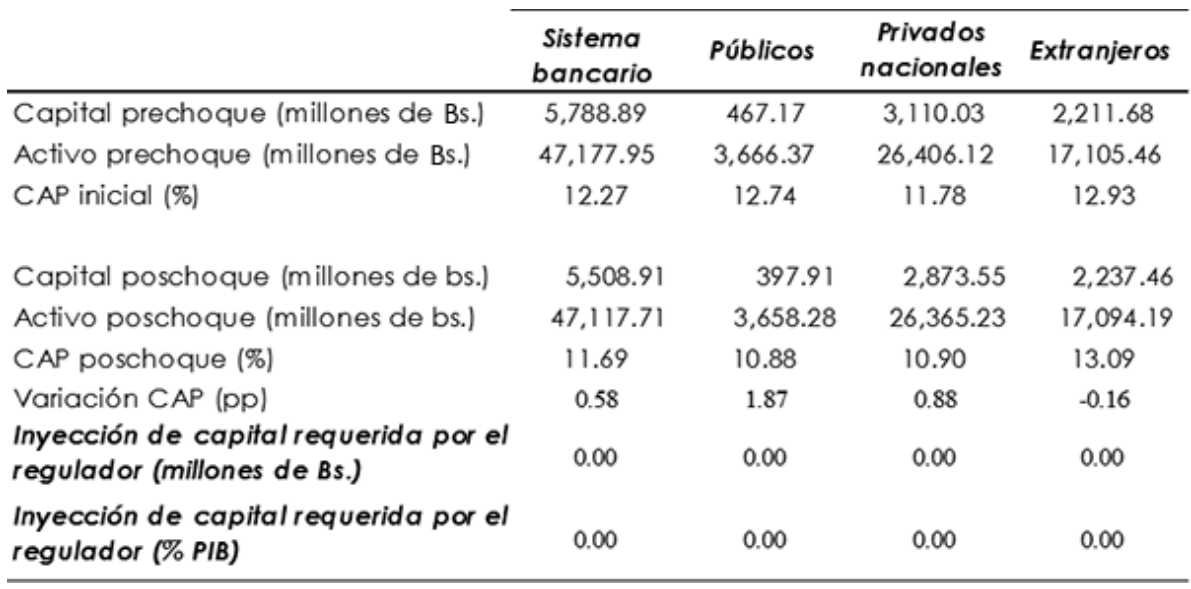

Nota: una variación negativa del CAP implica una capitalización del CAP.

\section{Conclusiones}

A partir de la evaluación integral de los cuatro módulos de riesgo (riesgo de crédito, de tasas de interés, cambiario, de liquidez) en los cuatro escenarios planteados, se concluye que el riesgo más importante para el sistema bancario boliviano es el de tasa de interés ${ }^{34}$. Esto a partir de la disparidad entre los descalces de plazos entre activos y pasivos en los bancos públicos (Banco Unión) y los bancos privados nacionales, mientras que los bancos extranjeros cuentan con un GAP positivo que los cobertura ante este riesgo.

En materia cambiaria, se destaca el efecto favorable que ejerce una depreciación del tipo de cambio en la capitalización bancaria, dada la posición neta larga (positiva) en moneda extranjera que posee el sistema bancario boliviano. Sin embargo, al contraponerse el efecto indirecto a este último, en términos globales una devaluación generaría un impacto negativo sobre el CAP.

34 De acuerdo a los resultados hallados al analizar los escenarios adversos, se deduce que el riesgo de tasas de interés es el más importante (de la disminución total del capital en los escenarios, entre $72 \%$ a $97.2 \%$ fue generado por este tipo de riesgo), seguido por el riesgo de crédito (impacto entre $2.9 \%$ a $19.4 \%$ ) y el riesgo de tipo de cambio (0\% a $8.3 \%$ ). 
Por su parte, el análisis de riesgo de crédito no constituye per se una vulnerabilidad al sistema bancario. Esto principalmente debido a que el nivel de pesadez del sistema bancario a 2011 se encuentra en 1.65\%, el mínimo registrado hasta ese momento; a su vez, el hecho de que en promedio los créditos bancarios estén sujetos a un colateral, disminuye las provisiones necesarias. Estos elementos influyen en la determinación de la nueva cartera en mora y su efecto en el CAP. El modelo de créditos morosos muestra una conexión positiva con una devaluación cambiaria y con la tasa de interés; así también se demuestra que opera el crecimiento del PIB como variable. Esto alerta el potencial riesgo de que la actividad económica se desacelere en un futuro.

Con respecto al riesgo de liquidez, se comprobó que bajo ninguno de los escenarios planteados, tanto con retiros proporcionales como diferenciados de acuerdo al tamaño de cada banco, se detectaron problemas asociados con este riesgo. Sin embargo, dado el tamaño del Banco Mercantil Santa Cruz, los resultados muestran que podría enfrentar problemas de liquidez al cabo de cinco días de retiros de depósitos, por lo cual sería pertinente tener un criterio de tamaño en función de su cartera, para medir los posibles retiros de liquidez que se puedan dar.

Por último, una posible extensión de este trabajo sería ver el riesgo de crédito a través de los tipos de créditos reconocidos por la ASFI, y ver el impacto que puedan tener nuevas regulaciones, como la que determina que los bancos deben colocar un $15 \%$ de créditos a actividades productivas. Otra extensión interesante, planteada por Kida (2008), implica generar un mecanismo que permita mostrar el efecto del deterioro generado por el escenario macro adverso mediante el ST, y transmitirlo nuevamente a la economía, vía PIB u otro indicador, para mostrar el impacto en la economía. En cuanto a la metodología econométrica utilizada, se podría mejorar las estimaciones con datos de panel, e incluir una medición de riesgo de contagio en el trabajo. 


\section{Referencias}

1. Autoridad de Supervisión del Sistema Financiero (ASFI) (ex Superintendencia de Bancos y Entidades Financieras) (2008). Guías para la gestión de riesgos. Bolivia.

2. ------- (2011). Anuario Estadístico. Bolivia.

3. - - ----- (2012). Recopilación de normas para Bancos y Entidades Financieras; Bolivia.

4. - ------- Datos publicados en su página web: www.asfi.gob.bo; Bolivia.

5. Banco Central de Bolivia (2011). Informe de Estabilidad Financiera correspondiente al segundo semestre del 2011.

6. $\quad$------- (2012). Informe de Estabilidad Financiera correspondiente al segundo semestre del 2012.

7. $\quad$-------- Datos publicados en su página web: $\underline{\text { www.bcb.gob.bo }}$

8. Bank for International Settlements (2008). Principios para la realización y supervisión de pruebas de tensión.

9. Blaschke, Winfrid; Jones, Matthew T.; Majnoni, Giovanni y Martínez Peria, Soledad (2001). Stress Testing of Financial Systems: An Overview of Issues, Methodologies, and FSAP Experiences. International Monetary Fund (IMF), Working Paper $\mathrm{N}^{\circ} 01 / 88$.

10. Borio, Claudio; Drehmann, Mathias y Tsatsaronis, Kostas (2012). Stress testing macro stress testing: does it live up to expectations? Bank for International Settelments (BIS) Working Paper N³69.

11. Cihak, M. (2004). Stress Testing: A Review of key Concepts. Czech National Bank Research and Policy Notes.

12. ------ (2007). Introduction to applied stress testing. International Monetary Fund (IMF), Working Paper, 2007.

13. Cihak, M.y Hermanek, J. (2005). Stress testing the Czech Banking System: Where are we? Where are we going? Czech National Bank Research and Policy Notes.

14. Comité de Supervisión Bancaria, Basilea II (2006). Convergencia internacional de medidas y normas de capital. Bank for International Settlements (BIS).

15. Delgado, Saurina (2004). Riesgo de crédito y dotaciones a insolvencias. Un análisis con variables macroeconómicas. Dirección General de Regulación, Banco de España. 
16. Díaz, O. (2012); Identificación de booms crediticios en América Latina. Gerencia de Entidades Financieras - Banco Central de Bolivia.

17. Enders, Walter (2004). Applied Econometric Time Series. (2da. Edición). Estados Unidos: Wiley \& Sons.

18. Hernández, María Fernanda; Valero, Juan José y Días, María Bernardette (2007). Perfil de riesgos del sistema bancario venezolano: aplicación de la metodología de stress testing. CEMLA.

19. Hoggarth, Glenn, Logan, Andrew y Zicchino, Lea (2004). Macro stress tests of UK. Banks. Bank of England.

20. Hull, J. (2002). Introducción a los mercados de opciones y futuros (4ta. Edición). Madrid: Prentice Hall.

21. Hurtado, Enrique; Villacorta, Omary Ferruz, Luis (2008). La extranjerización del sistema bancario de Bolivia en la última década. Gestión Joven. Revista de la Agrupación Joven Iberoamericana de Contabilidad y Administración de Empresas. № 1.

22. Instituto Nacional de Estadística (INE). Datos publicados en su página web: www. ine.gob.bo.

23. Johansen, Soren (1988). Statistical Analysis of Cointegration Vectors. Journal of Economic Dynamics and Control, 12 (2-3), 231-254.

24. Jones, Matthew; Hilbers T. Paul y Slack, Graham (2004). Stress Testing the Financial Systems: What to Do When the Governor Calls. International Monetary Fund (IMF). Working Paper $\mathrm{N}^{\circ} 127$.

25. Mizuho Kida, (2008). Macro stress testing model with feedback effects. Central Bank of New Zealand.

26. McLachlan, G. J. (2008). Mahalanobis Distance. Indian Academy of Sciences.

27. Sheriff, Ernesto (2010). Inflationary memory as restrictive factor of the impact of the public expense in the economic growth: lessons from high inflation Latin American countries using an innovative inflationary memory indicator. Instituto de Estudios Avanzados en Desarrollo (INESAD). Working Paper. 


\section{Anexo 1}

Especificación de los modelos y análisis de cointegración

\begin{tabular}{|c|c|c|c|}
\hline \multicolumn{2}{|c|}{$\begin{array}{c}\text { Modelo VEC - Riesgo de } \\
\text { liquidez }\end{array}$} & \multicolumn{2}{|c|}{$\begin{array}{c}\text { Modelo VEC - Riesgo de } \\
\text { crédito }\end{array}$} \\
\hline V ariables & Coeficientes & Variables & Coeficientes \\
\hline \multirow{3}{*}{$\lambda$} & -0.053926 & & -0.063056 \\
\hline & $(0.01739)$ & $\lambda$ & $(0.02950)$ \\
\hline & {$[-3.10007]$} & & {$[-2.13775]$} \\
\hline \multirow[t]{2}{*}{ LOGDEPO(-1)* } & 1.000000 & LOGMORA_SA(-1)* & 1.000000 \\
\hline & 17.17566 & & 10.54684 \\
\hline \multirow[t]{3}{*}{$\operatorname{DEV}(-1)$} & $(8.25523)$ & GCARTERA(-1) & $(1.62245)$ \\
\hline & [ 2.08058] & & [ 6.50056] \\
\hline & -2.28341 & & -8.832226 \\
\hline \multirow[t]{3}{*}{ LOGPIBCONS_SA $(-1)$} & $(0.34409)$ & $\operatorname{DEV}(-1)$ & $(5.71916)$ \\
\hline & {$[-6.63609]$} & & {$[-1.74432]$} \\
\hline & 0.260974 & & -1.113877 \\
\hline \multirow[t]{3}{*}{ LOGTASA_PAS(-1) } & $(0.08178)$ & LOGTASA_ACT(-1) & $(0.38197)$ \\
\hline & [ 3.19111$]$ & & {$[-2.91615]$} \\
\hline & 1.196974 & & 0.282133 \\
\hline \multirow[t]{2}{*}{ LOGIPC(-1) } & $(0.41756)$ & GPIB(-1) & $(0.04678)$ \\
\hline & [ 2.86662] & & [6.03124] \\
\hline $\mathrm{C}$ & -2.875823 & $\mathrm{C}$ & -3.9164 \\
\hline R-squared & 0.409988 & R-squared & 0.564519 \\
\hline
\end{tabular}

Nota. En paréntesis se muestra la desviación estándar y en corchetes el estadístico t. La interpretación de los coeficientes es opuesta al signo. 


\begin{tabular}{ccccc}
\hline \multicolumn{5}{c}{ Modelo VEC - Riesgo de crédito } \\
\hline Ho & $\lambda$ trace & Prob. & $\lambda$ max & Prob. \\
\hline None * & 97.49 & 0.00 & 53.42 & 0.00 \\
At most 1 & 44.1 & 0.11 & 23.54 & 0.15 \\
At most 2 & 20.53 & 0.39 & 15.20 & 0.28 \\
At most 3 & 5.33 & 0.77 & 4.35 & 0.82 \\
At most 4 & 0.98 & 0.32 & 0.98 & 0.32 \\
\hline \multicolumn{5}{c}{ Modelo VEC - Riesgo de liquidez } \\
\hline Ho & $\lambda$ trace & Prob. & $\lambda$ max & Prob. \\
\hline None * & 97.38 & 0.00 & 44.59 & 0.00 \\
At most 1 & 52.80 & 0.02 & 32.59 & 0.01 \\
At most 2 & 20.21 & 0.41 & 9.89 & 0.75 \\
At most 3 & 10.32 & 0.26 & 8.61 & 0.32 \\
At most 4 & 1.71 & 0.19 & 1.71 & 0.19 \\
\hline
\end{tabular}

Nota. Las estimaciones incluyen los rezagos 1, 3, 5, 8 (sólo para el VEC - riesgo de crédito) y una constante 


\section{Anexo 2}

Escenario: Inestabilidad política (en millones de bolivianos y porcentajes)

\begin{tabular}{|c|c|c|c|c|}
\hline & \\
\hline & $\begin{array}{c}\text { Sistema } \\
\text { bancario }\end{array}$ & Públicos & $\begin{array}{c}\text { Privados } \\
\text { nacionales }\end{array}$ & Extranjeros \\
\hline Capital prechoque (millones de Bs.) & $5,788.89$ & 467.17 & $3,110.03$ & $2,211.68$ \\
\hline Activo prechoque (millones de Bs.) & $47,177.95$ & $3,666.37$ & $26,406.12$ & $17,105.46$ \\
\hline CAP inicial $(\%)$ & 12.27 & 12.74 & 11.78 & 12.93 \\
\hline Capital poschoque (millones de bs.) & $4,831.75$ & 188.00 & $2,232.54$ & $2,411.21$ \\
\hline Activo poschoque (millones de bs.) & $47,141.18$ & $3,661.43$ & $26,381.16$ & $17,098.58$ \\
\hline CAP poschoque (\%) & 10.25 & 5.13 & 8.46 & 14.10 \\
\hline Variación CAP (pp) & 2.02 & 7.61 & 3.32 & -1.17 \\
\hline $\begin{array}{l}\text { Inyección de capital requerida por el } \\
\text { regulador (millones de Bs.) }\end{array}$ & 647.81 & 533.78 & 114.02 & 0.00 \\
\hline $\begin{array}{l}\text { Inyección de capital requerida por el } \\
\text { regulador (\% PIB) }\end{array}$ & 0.47 & 0.39 & 0.08 & 0.00 \\
\hline
\end{tabular}

Escenario: Shock de créditos (en millones de bolivianos y porcentajes)

\begin{tabular}{lcccc} 
& $\begin{array}{c}\text { Sistema } \\
\text { bancario }\end{array}$ & Públicos & $\begin{array}{c}\text { Privados } \\
\text { nacionales }\end{array}$ & Extranjeros \\
\hline Capital prechoque (millones de Bs.) & $5,788.89$ & 467.17 & $3,110.03$ & $2,211.68$ \\
Activo prechoque (millones de Bs.) & $47,177.95$ & $3,666.37$ & $26,406.12$ & $17,105.46$ \\
CAP inicial (\%) & 12.27 & 12.74 & 11.78 & 12.93 \\
& & & & \\
Capital poschoque (millones de bs.) & $4,795.39$ & 176.09 & $2,197.12$ & $2,422.18$ \\
Activo poschoque (millones de bs.) & $47,145.55$ & $3,662.02$ & $26,384.13$ & $17,099.40$ \\
CAP poschoque (\%) & 10.17 & 4.81 & 8.33 & 14.17 \\
$\begin{array}{l}\text { Variación CAP (pp) } \\
\text { Inyección de capital requerida por el }\end{array}$ & 2.10 & 7.93 & 3.45 & -1.24 \\
regulador (millones de Bs.) & 696.57 & 570.71 & 125.86 & 0.00 \\
$\begin{array}{l}\text { Inyección de capital requerida por el } \\
\text { regulador (\% PIB) }\end{array}$ & 0.50 & 0.41 & 0.09 & 0.00 \\
\hline
\end{tabular}


Escenario: Shock de subida del encaje legal (en millones de bolivianos y porcentajes)

\begin{tabular}{lcccc} 
& $\begin{array}{c}\text { Sistema } \\
\text { bancario }\end{array}$ & Públicos & $\begin{array}{c}\text { Privados } \\
\text { nacionales }\end{array}$ & Extranjeros \\
\hline Capital prechoque (millones de Bs.) & $5,788.89$ & 467.17 & $3,110.03$ & $2,211.68$ \\
Activo prechoque (millones de Bs.) & $47,177.95$ & $3,666.37$ & $26,406.12$ & $17,105.46$ \\
CAP inicial (\%) & 12.27 & 12.74 & 11.78 & 12.93 \\
& & & & \\
Capital poschoque (millones de bs.) & $5,090.64$ & 262.69 & $2,468.59$ & $2,359.36$ \\
$\begin{array}{l}\text { Activo poschoque (millones de bs.) } \\
\text { CAP poschoque (\%) }\end{array}$ & $47,155.18$ & $3,663.32$ & $26,390.66$ & $17,101.20$ \\
$\begin{array}{l}\text { Variación CAP (pp) } \\
\text { Inyección de capital requerida por el }\end{array}$ & 10.80 & 7.17 & 9.35 & 13.80 \\
regulador (millones de Bs.) & 334.63 & 5.57 & 2.42 & -0.87 \\
$\begin{array}{l}\text { Inyección de capital requerida por el } \\
\text { regulador (\% PIB) }\end{array}$ & 0.50 & 294.81 & 398.20 & 0.00 \\
\hline
\end{tabular}

Nota: una variación negativa del CAP implica una capitalización del CAP. 